\title{
Antiparasitic activity in Asteraceae with special attention to ethnobotanical use by the tribes of Odisha, India
}

\author{
Sujogya Kumar Panda ${ }^{1,2, *}$ and Walter Luyten ${ }^{2}$ \\ ${ }^{1}$ Department of Zoology, North Orissa University, Baripada-757003, India \\ ${ }^{2}$ Department of Biology, KU Leuven, 3000 Leuven, Belgium
}

Received 6 November 2017, Accepted 3 February 2018, Published online 12 March 2018

\begin{abstract}
The purpose of this review is to survey the antiparasitic plants of the Asteraceae family and their applicability in the treatment of parasites. This review is divided into three major parts: (a) literature on traditional uses of Asteraceae plants for the treatment of parasites; (b) description of the major classes of chemical compounds from Asteraceae and their antiparasitic effects; and (c) antiparasitic activity with special reference to flavonoids and terpenoids. This review provides detailed information on the reported Asteraceae plant extracts found throughout the world and on isolated secondary metabolites that can inhibit protozoan parasites such as Plasmodium, Trypanosoma, Leishmania, and intestinal worms. Additionally, special attention is given to the Asteraceae plants of Odisha, used by the tribes of the area as antiparasitics. These plants are compared to the same plants used traditionally in other regions. Finally, we provide information on which plants identified in Odisha, India and related compounds show promise for the development of new drugs against parasitic diseases. For most of the plants discussed in this review, the active compounds still need to be isolated and tested further.
\end{abstract}

Keywords: Asteraceae, Plasmodium, Trypanosoma, Leishmania, Odisha (India), antiparasitic drugs

Résumé - Activité antiparasitaire chez les Asteraceae avec une attention particulière pour l'utilisation ethnobotanique par les tribus d'Odisha en Inde. Le but de cette revue est d'étudier les plantes antiparasitaires de la famille des Asteraceae et leur applicabilité dans le traitement des parasites. Cette revue est divisée en trois parties principales: (a) littérature sur les utilisations traditionnelles des Asteraceae pour le traitement des parasites; (b) description des principales classes de composés chimiques des Asteraceae et leurs effets antiparasitaires; (c) activité antiparasitaire avec référence spéciale aux flavonoïdes et aux terpénoïdes. Cette revue fournit des informations détaillées sur les extraits d'Asteraceae rapportés à travers le monde et sur des métabolites secondaires isolés qui peuvent inhiber les parasites protozoaires, tels que Plasmodium, Trypanosoma, Leishmania et les vers intestinaux. En outre, une attention particulière est accordée aux Asteraceae d'Odisha (Inde), utilisées par les tribus locales comme antiparasitaires. Ces plantes sont comparées aux mêmes espèces utilisées traditionnellement dans d'autres régions. Enfin, nous fournissons des informations sur les plantes identifiées à Odisha et les composés qui seraient prometteurs en tant que médicaments candidats contre les maladies parasitaires. Pour la plupart des plantes discutées dans cette revue, les composés actifs doivent encore être isolés et testés plus avant.

\section{Introduction - Antiparasitic research}

Parasite diseases are a major source of disease in both humans and animals and result in significant economic losses. Protozoan parasites threaten the lives of billions of

\footnotetext{
*Corresponding author: suju.panda@kuleuven.be; sujogyapanda@gmail.com
}

people worldwide and are associated with significant morbidity and large economic impacts [88]. The lack of proper vaccines and the emergence of drug resistance make the search for new drugs for treatment and prophylaxis more urgent, including from alternative sources like plants. In 2005, Pink et al. published a review emphasizing that new antiparasitic drugs are urgently needed to treat and control diseases such as malaria, 
leishmaniasis, sleeping sickness and filariasis [124]. The discovery of quinine from Cinchona succirubra (Rubiaceae) and its subsequent development as an antimalarial drug represent a milestone in the history of antiparasitic drugs from nature. The 2015 Nobel Prize in Physiology or Medicine was awarded for the discovery of artemisinin and avermectin, which fundamentally changed the treatment of parasitic diseases around the globe. Both compounds are natural products, once again showing that nature can be a powerful source of medicines. A breakthrough for the development of antimalarial drugs was the identification of the sesquiterpene artemisinin from Artemisia annua (Asteraceae), which can even kill multidrug-resistant strains of Plasmodium falciparum $[3,62]$. Several semisynthetic derivatives of artemisinin (e.g., the water-soluble artesunate) have been developed and are used in clinical practice today [62].

There are three major protozoan parasitic infections, caused by Plasmodium, Leishmania and Trypanosoma species. Plasmodium is the most significant of the protozoan parasites that infect humans. Found in tropical and sub-tropical regions of the world, malaria parasites threaten the lives of 3.3 billion people and cause $0.6-$ 1.1 million deaths annually [70]. Six species of Plasmodium are responsible for causing malaria in humans [144], with Plasmodium falciparum and Plasmodium vivax being the most common and major causes. Leishmaniasis is caused by Leishmania sp., generating 1-1.5 million new cases annually [104]. The disease is endemic in 98 countries and is one of the neglected tropical diseases where the majority of the affected individuals are rural, underprivileged, and economically disadvantaged. African sleeping sickness (trypanosomiasis), is caused by two parasitic protozoans: Trypanosoma brucei gambiense (West Africa) and Trypanosoma brucei rhodesiense (East Africa) [15]. African trypanosomiasis threatens the lives of approximately 60 million people in sub-Saharan Africa and is fatal if untreated [70]. Another species of Trypanosoma (T. cruzi) is responsible for Chagas disease (American trypanosomiasis), and threatens the lives of millions primarily in Mexico, Latin America and the United States. The World Health Organization estimates that 810 million people are infected annually. There is also no vaccine for Chagas disease and no clinical trials of new drugs are under way; current treatment depends on only two chemotherapeutics - benznidazole and nifurtimox.

\section{Medicinal uses of Asteraceae with special reference to the tribes of Odisha (Orissa), India}

The family Asteraceae (Compositae) is also known as the daisy family, sunflower family or thistle family. Asteraceae is derived from the term "aster" meaning "star" in Latin, and refers to the characteristic inflorescence with flower heads composed of florets (small flowers), and surrounded by bracts [12]. The family Asteraceae is one of the largest families comprising 1600-1700 genera and
24,000-30,000 species [30]. The family has 12 subfamilies and 43 tribes, and is distributed worldwide [16], but is most abundant in the temperate and warm-temperate regions. Most of the species are herbs and shrubs, while trees are fewer in number. Asteraceae have been commonly used in the treatment of various diseases since ancient times, as attested by classical literature. For this review, we collected literature from scientific journals, books, theses and reports via a library and electronic search (using databases viz. PubMed, Google Scholar and Scopus). Several researchers have systematically investigated Asteraceae for their therapeutic utility. More than 7000 compounds have already been isolated, and 5000 have been identified from this family, often associated with some bioactivity [3]. Members of the Asteraceae are claimed to have various properties: antipyretic, antiinflammatory, detoxifying, antibacterial, wound-healing, antihemorrhagic, antalgic (also for headaches), antispasmodic, and anti-tussive, and have been considered beneficial for flatulence, dyspepsia, dysentery, lumbago, leucorrhoea, hemorrhoids, hypotension, and most importantly, some are hepatoprotective, antitumor and antiparasitic [68]. The majority of studies on Asteraceae throughout the world have focused on chemical analysis (nearly 7000 compounds already isolated). There are many papers on in vitro studies, especially on antimicrobial, antioxidant and anticarcinogenic properties, using selected cells and crude extracts or purified compounds. In the few published reviews on pure compounds, the structure-activity relations were studied as well as their mechanism of action. Despite the discovery of a large number of compounds in Asteraceae around the world, and the reported antiparasitic properties of members of the Asteraceae family, not many bioactivity studies on Asteraceae species have yet been carried out. In India, the family is represented by 900 species from 167 genera.

Due to their bioactive properties, plants from the Asteraceae family are commonly used in the traditional treatment of various diseases (Table 1). For instance, Ageratum conyzoides has been commonly used in India including in the state of Odisha, where the plant is traditionally used for diarrhoea, dysentery, intestinal colic [118] and malaria. This plant is well-known for the presence of phytochemicals such as alkaloids, coumarins, flavonoids, benzofurans, sterols and terpenoids, with the following identified compounds: friedelin, various sterols (including $\beta$-sitosterol and stigmasterol), various flavonoids, caryophyllene, coumarin, quercetin, as well as fumaric and caffeic acid [51]. Bidens pilosa is also found in Odisha, and is moreover widely used as folk medicine by indigenous tribes of the Amazon in the treatment of malaria [13]. About 201 compounds comprising 70 aliphatics, 60 flavonoids, 25 terpenoids, 19 phenylpropanoids, 13 aromatics, 8 porphyrins, and 6 other compounds, have been identified from this plant, as compiled previously [67]. However, the relation between Bidens pilosa phytochemicals and various bioactivities is not yet fully established, and should become a future research focus [7]. Blumea lacera is used for the treatment 
Table 1. Traditional uses of plants of the Asteraceae family

\begin{tabular}{lll}
\hline Plant $^{1}$ & Traditional uses by the tribes of Odisha & Other parts of India/world \\
\hline Ageratum conyzoides (L.) L. & $\begin{array}{l}\text { Herb infusion is given for gastrointestinal } \\
\text { ailments such as diarrhoea, dysentery and }\end{array}$ & As worm medicine in Cameroon [157]. \\
& intestinal colic with flatulence [117,120]. Cold \\
& decoctions from the aerial parts are used to cure \\
& malarial fever (unpublished observations). &
\end{tabular}

Bidens pilosa L.

Blumea lacera (Burm.f.) DC. The tribes use fresh leaf juice of this plant for

Calendula officinalis L.

Caesulia axillaris Roxb.

Centipeda minima (L.)

A. Braun \& Asch.

Eclipta alba (L.) Hassk.

Eclipta prostrata (L.) L. is a synonym of Eclipta alba (L.) Hassk.

Elephantopus scaber L.

Sphaeranthus indicus L.

Tagetes erecta $\mathrm{L}$.

Tridax procumbens (L.) L. the treatment of all kinds of fever, including malaria (unpublished observations).

Fresh juice from the aerial parts is used for intestinal worm infections, abdominal pain and stomach ache (unpublished observations).

Cold decoction of leaf is used for amoebic and bloody dysentery (unpublished observations).

Whole plant extract is given to cure malaria [113].

Root decoction is used for the treatment of all kinds of fever [112]. Leaf decoction is commonly used for hookworm and roundworm (unpublished observations).

Treatment of malaria [112].

Treatment of malaria: decoction of dried leaf with tea leaf tincture is administered orally twice a day for five days [118].

Treatment of malaria: paste prepared from fresh root is taken orally once a day for three days [118]. Juice of leaf is used in the treatment of malaria [53].

Helminths: whole plant paste with a pinch of salt is taken as an anthelmintic [107].

Cold decoctions of leaf and flower are used for all kinds of worm infections and dysentery (unpublished observations).

Decoction prepared from leaves of Tridax procumbens and Andrographis paniculata (Burm. f.) Nees is used for the treatment of malaria fever (unpublished observations).
Juice form the root and whole plant is used for the treatment of malaria (Africa, China) $[142,157]$. Whole plant is used by the Bukusu community of Kenya for tick prevention and control on livestock [159].

Leaf juice is used to kill worms in children by the tribes of Madhya Pradesh, India [136].

Flowers are used for the treatment of intestinal worms and amoebal infections in pets and pigs in British Columbia, Canada [64].

The whole plant is crushed and juice is extracted, which is given orally three times a day, along with curd to cure amoebic dysentery by the tribes of Madhya Pradesh, India [155].

In China, decoction from whole plant is used for malaria treatment. The seed or dried aerial parts are used as a vermifuge and amoebicide (http://uses.plantnet-project.org/ en/Centipeda_minima_(PROSEA).

Leaf decoction is used by the Rakhain tribal healers of Chittagong Division, Bangladesh for the treatment of malaria [46].

Infusion or juice of the plant mixed with honey is given for the treatment of malaria by the tribal communities of Pakistan [86].

Decoction from aerial parts is used to treat malaria by the tribes of Madagascar [86].

Root and seed powder is given orally to kill intestinal worms in children [39]. Whole plant paste with a pinch of common salt is taken as an anthelmintic [61].

Plants used by native Amazonian groups from the Nanay River (Peru) for the treatment of malaria [61].

Used for the treatment of malaria by the tribes of Ghana [59], and Kwale community of the Kenyan Coast [90]. 
Table 1. (continued).

\begin{tabular}{ll}
\hline Plant $^{1}$ & Traditional uses by the tribes of Odisha \\
\hline $\begin{array}{l}\text { Vernonia anthelmintica (L.) } \\
\text { Willd. This name is a }\end{array}$ & $\begin{array}{l}\text { Fruit powder is used in malaria fever, and for } \\
\text { stomach ache during amoebic dysentery [81]. }\end{array}$ \\
$\begin{array}{l}\text { synonym of Baccharoides } \\
\text { anthelmintica } \text { (L.) Moench. } \\
\text { anthelminticum (L.) Kuntze }\end{array}$ & $\begin{array}{l}\text { Seeds are used as an anthelmintic, especially in } \\
\text { children }(2-5 \mathrm{~g} \text { with water on an empty stomach } \\
\text { twice a day for three days) [111,112]. }\end{array}$ \\
$\begin{array}{ll}\text { Vernonia albicans } \text { DC. } \\
\begin{array}{l}\text { This name is a synonym of } \\
\text { Cyanthillium albicans (DC.) }\end{array}\end{array}$ & $\begin{array}{l}\text { Filariasis: powdered plant (10-20 g) is advised to } \\
\text { be consumed with } 125 \mathrm{~mL} \text { milk (mixed with }\end{array}$ \\
$\begin{array}{l}\text { 5-7 cardamom fruits and } 10 \mathrm{~g} \text { sugar candy) once } \\
\text { daily in the morning, on an empty stomach, for } \\
\text { about three months [37]. Water-extract of the } \\
\text { whole plant is used in the treatment of malaria [53]. }\end{array}$
\end{tabular}

Vernonia cinerea (L.) Less. This name is a synonym of Cyanthillium cinereum (L.) H. Rob.

Xanthium strumarium L.
Treatment of malaria; root paste is mixed with honey and administered orally twice a day for three days [118]. The plant is also used for elephantiasis [120].

Coastal tribes of Odisha use crushed fresh fruit for the treatment of filariasis (unpublished observations)

Other parts of India/world

The seeds are used as an anthelmintic against parasitic worm (including tapeworm) infestations [4].

Leaf and bark are used by the tribes of Equatorial Guinea as febrifuge and vermifuge [2], while the tribes of Tanzania use it for the treatment of malaria [84].

Tribes of Bannu district, Pakistan, use it for the treatment of chronic malaria [154].

${ }^{1}$ All taxonomic names were verified in the Global Composite Checklist database (http://compositae.landcareresearch.co.nz/Default.aspx)

of all kinds of fever, including malaria, and contains phytocompounds such as fenchone, coniferyl alcohol derivatives, campesterol, flavonoids, lupeol, hentriacontane, hentriacontane, $\alpha$-amyrin, $\beta$-sitosterol and triterpenes $[7,80,105]$. Calendula officinalis has found many medicinal applications and contains various terpenoids (sitosterols, stigmasterols, erythrodiol, brein, ursadiol and its derivatives; several triterpene glycosides like calendulaglycoside A; glucosides of oleanolic acid, etc.), various flavonoids (quercetin, isoquercetin, isorhamnetin-3-O$\beta$-D-glycoside, narcissin, calendoflaside, calendoflavoside, calendoflavobioside, rutin, quercetin-3-O-glucoside and quercetin-3-O-rutinoside), coumarins, saponins and quinones [87].

Whole plant extracts of Caesulia axillaris are frequently used by the coastal tribes of Odisha to cure malaria $[107,113]$, but no scientific studies have yet been published on this plant. Centipeda minima is widely distributed in Odisha, and is frequently used by the local tribes for the treatment of parasites [112], but no compounds responsible for its antiparasitic activities have yet been identified. Eclipta prostrata (synonym E. alba) is frequently used by the tribes for the treatment of malaria $[113,130]$. The plant is well studied for its phytochemistry, with documented presence of compounds such as eclipline, $\beta$-amyrin, luteolin-7-O-glucoside, apigenin, cinnaroside, stigmasterol, wedelolactone, columbin, triterpene glycosides and triterpenic acid [47]. Like Eclipta prostrata, Elephantopus scaber is also frequently used by the tribes for the treatment of malaria [118]. This plant is also well studied for its phytochemistry with documented presence of sesquiterpenelactones such as elescaberin, deoxyelephan- topin, isodeoxyelephantopin, scabertopin, and isoscabertopin, and lipids like ethyl hexadecanoate, ethyl-9, 12-octadecadienoate, ethyl-(Z)-9-octadecenoate, ethyl octadecanoate, lupeol and stigmasterol [19]. Whole plant paste of Sphaeranthus indicus with a pinch of salt is taken as an anthelmintic by the tribes of Odisha [111]. The phytochemical studies of this plant suggest the presence of eudesmanolides, sesquiterpenoids, sesquiterpene lactones, sesquiterpene acids, flavone glycosides, flavonoid C-glycosides, isoflavone glycosides, sterols, sterol glycosides, alkaloids, peptide alkaloids, amino acids and sugars [125]. The essential oil from this plant has been well studied with the documented presence of bioactive compounds like sphaeranthine, sphaeranthol, spharerne, methyl chavicol, ocimene, geraniol, and methoxy frullanolides [71]. Tagetes erecta is an ornamental plant of Odisha and is often used by the tribes for the treatment of various conditions such as anaemia, irregular menstruation, abdominal pain, colic, cough and dysentery. Like Sphaeranthus indicus, this plant is also well known for its phytoconstituents such as $\beta$-sitosterol, $\beta$-daucosterol, 7 hydroxy sitosterol, lupeol, erythrodiol, erythrodiol-3palmitate, quercetagetin, quercetagetin-7-methyl ether, quercetagetin-7-O-glucoside, gallic acid, syringic acid, quercetin, ocimene and tagetone [135]. Tridax procumbens has been extensively used in Ayurvedic medicine and is well-studied for its phytochemistry, with the presence of compounds like 8,3'-dihydroxy-3,7,4'-trimethoxy-6-O$\beta-\mathrm{D}$ glucopyranoside flavonol, apigenin-7-O- $\beta$-D-glucoside, pentadecane, $\beta$-sitosterol, stigmasterol, $\beta$-daucesosterol and bis-(2-ethylhexyl)-phthalate [131]. Several species of Vernonia have been used in different traditional 
medicines all over the world. The tribes of Odisha most frequently use different species of Vernonia: $V$. anthelmintica, V. albicans and $V$. cinerea. Seeds of Vernonia anthelmintica are used as an anthelmintic, especially in children: $2-5 \mathrm{~g}$ with water on an empty stomach twice a day for three days [111,112]. Fruit powder is used in malaria fever, and stomach ache during amoebic dysentery [81]. Powdered Vernonia albicans plant (10-20 g) is advised to be consumed with $125 \mathrm{~mL}$ milk (mixed with 5 7 cardamom fruits and $10 \mathrm{~g}$ sugar candy) once in the morning, on an empty stomach for about three months for the treatment of filariasis [37]. The aqueous extract of the whole plant is also used in the treatment of malaria [53]. Root paste of Vernonia cinerea mixed with honey is administered orally twice a day for three days for malaria [108]. Reports are also available on the use of this plant for the treatment of elephantiasis [108]. Toyang and Verpoorte [152] published a review article on this genus Vernonia (109 species) concerning its ethnopharmacology and phytochemistry. Xanthium strumarium is a weed, widely distributed in Odisha, and commonly used as a medicinal plant. Most of its pharmacological effects can be explained by constituents like sesquiterpene lactones, glycosides, phenols, as well as polysterols present in all plant parts. The bioactive compounds reported for this plants are xanthinin, xanthumin, xanthatin (deacetylxanthinin), a toxic principle, namely a sulphated glycoside: xanthostrumarin, atractyloside, carboxyatractyloside, phytosterols, xanthanol, isoxanthanol, xanthinosin, 4-oxo-bedfordia acid, hydroquinone, xanthanolides, caffeoylquinic acids, $\alpha$ - and $\gamma$-tocopherol, thiazinedione and deacetyl xanthumin, $\beta$-sitosterol, $\gamma$-sitosterol, $\beta$-D-glucoside of $\beta$-sitosterol; isohexacosane, chlorobutanol, stearyl alcohol, stromasterol and oleic acid [52].

\section{Miscellaneous antiparasitic properties of Asteraceae and their phytochemistry}

Over the past decades, a lot of research on antiparasitic drugs of plant origin has yielded undisputable metabolites of interest. Many plant-derived secondary metabolites of Asteraceae have exhibited target-specific activity against Plasmodium, Leishmania and Trypanosoma parasites (Table 2). Plants from the Asteraceae family are widely used as medicines due to the presence of a broad range of bioactive metabolites such as alkaloids (pyrrolizidine and pyridine), flavonoids, phenolic acids, coumarins, terpenoids (monoterpenes, sesquiterpenes, diterpenes, and triterpenes), quinoline and diterpenoid types, triterpenoid sesquiterpene lactones, pyrethrins, and saponins. Several sesquiterpenes have been reported as antiprotozoal since the discovery of artemisinin. The sesquiterpene lactone parthenin is effective against Plasmodium falciparum in vitro, with an $\mathrm{EC}_{50}$ value of $1.29 \mu \mathrm{g} / \mathrm{mL}$ [123]. Parthenin is capable of blocking parasite-specific targets responsible for glutathinonylspermidine and trypanothione synthesis from cysteine and glutathione precursors in both Leishmania and Trypanosoma [32]. The sesquiterpene lactones brevilin A from Centipeda minima and dehydrozaluzanin C from Munnozia maronii were discovered and reported as antiparasitic. Similarly, sesquiterpene lactones from Neuroleaena lobata are well established for the treatment of Plasmodium infections [28]. In this plant, structureactivity relationship analysis revealed that germanocrenolide sesquiterpenes, like neurolenin $\mathrm{A}\left(\mathrm{EC}_{50}=0.92 \mu \mathrm{M}\right)$ and $\mathrm{B}\left(\mathrm{EC}_{50}=0.62 \mu \mathrm{M}\right)$, were more potent than furanoheliangolides like lobatin $\mathrm{A}$ and $\mathrm{B}\left(\mathrm{EC}_{50}=15.62 \mu \mathrm{M}\right.$ and $16.51 \mu \mathrm{M})$, respectively, against Leishmania promastigotes and Trypanosoma epimastigotes [28]. Based on ethnozoological studies (wild chimpanzees were observed to chew young stems of Vernonia amygdalina), antiplasmodial sesquiterpenes vernodalin and vernolide, hydroxyverniladin have been isolated [60]. Oketch-Rabah et al. [101] observed that macrocyclic germancrane dilactone 16,17-dihydrobrachycalyxolide from Vernonia brachycalyx has both antileishmanial and antiplasmodial activity.

Phenols are widely distributed in Asteraceae, and some have the ability to inhibit parasites. Gallic acid and its derivatives inhibit the proliferation of Trypanosoma cruzi trypomastigotes in vitro [58]. Higher activities were observed for the gallic acid esters ethyl-gallate and npropyl-gallate, which had $\mathrm{EC}_{50}$ values of 2.28 and $1.47 \mu \mathrm{g} /$ $\mathrm{mL}$, respectively, possibly due to increased lipophilicity. Oketch-Rabah et al. [101] reported the antiprotozoal activity from Vernonia brachycalyx (2́-epicycloisobrachycoumarinone epoxide and its stereoisomer). Both stereoisomers show similar in vitro activities against chloroquine-sensitive (CQ-S) and chloroquine-resistant (CQ-R) strains for Plasmodium falciparum, as well as Leishmania major promastigotes, with $\mathrm{EC}_{50}$ values of $0.11 \mu \mathrm{g} / \mathrm{mL}$ and $0.15 \mu \mathrm{g} / \mathrm{mL}$ for Plasmodium falciparum, and $37.1 \mu \mathrm{g} / \mathrm{mL}$ and $39.2 \mu \mathrm{g} / \mathrm{mL}$ for Leishmania major, respectively. Like phenols, flavonoids are extensively present in Asteraceae plants. Elford et al. [21] demonstrated that methoxylated flavonones artemetin and casticin act synergistically with artemisinin in vitro against Plasmodium falciparum. Later, exiguaflavanone A and B, isolated from Artemisia indica (Asteraceae), were shown to exhibit in vitro activity against Plasmodium falciparum.

The flavonoids can be classified into several subtypes: flavone (1), flavonol (2), flavanone (3), dihydroflavonol (4), flavan-3-ol (5), flavan-3,4-diol (6), chalcone (a structure with one opened ring), aurone, and anthocyanidine (with a positive charge on oxygen O-1). Except for these basic structures, flavonoids also exist in biflavonoid and glycosidic form in the Asteraceae family. PerezVictoria et al. [122] suggested that flavonoids could affect transport mechanisms in Leishmania. The C-terminal nucleotide-binding domain of a P-glycoprotein-like transporter, encoded by the ltrmdr1 gene in Leishmania tropica and involved in parasite multidrug resistance (MDR), was overexpressed in Escherichia coli as a hexahistidinetagged protein and purified. The Leishmania tropica recombinant domain efficiently bound different classes of flavonoids with the following relative affinity: flavone>- 
Table 2. Therapeutic uses of important plants of the Asteraceae family reported as an antiparasitic

\begin{tabular}{|c|c|c|c|c|c|c|}
\hline Plant $^{1}$ & $\begin{array}{l}\text { Plant part } \\
\text { used }\end{array}$ & Pharmacological & Preparation & Organism tested & $\begin{array}{l}\text { Context } \\
\text { of use }\end{array}$ & Reference \\
\hline \multirow[t]{2}{*}{$\begin{array}{l}\text { Acanthospermum } \\
\text { hispidum DC. }\end{array}$} & Whole plant & Antileishmanial & Ethanol extract & $\begin{array}{l}\text { Leishmania amazonensis } \\
\text { Leishmania braziliensis }\end{array}$ & In vitro & {$[27]$} \\
\hline & Aerial part & Antitrypanosomal & $\begin{array}{l}\text { Dichloromethane/ } \\
\text { Methanol/ Aqueous }\end{array}$ & $\begin{array}{l}\text { Trypanosoma brucei } \\
\text { brucei }\end{array}$ & In vitro & {$[10]$} \\
\hline $\begin{array}{l}\text { Achyrocline flaccida } \\
\text { (Weinm.) DC. }\end{array}$ & Whole plant & Antileishmanial & Ethanol extract & Leishmania amazonensis & In vitro & {$[27]$} \\
\hline $\begin{array}{l}\text { Ageratina } \\
\text { pentlandiana (DC.) } \\
\text { R. M. King \& H. Rob. }\end{array}$ & Leaf & Antileishmanial & Ethanol extract & $\begin{array}{l}\text { Leishmania amazonensis } \\
\text { Leishmania braziliensis }\end{array}$ & $\begin{array}{l}\text { In vitro } \\
\text { In vitro }\end{array}$ & $\begin{array}{l}{[27]} \\
{[69]}\end{array}$ \\
\hline \multirow[t]{5}{*}{$\begin{array}{l}\text { Ageratum } \\
\text { conyzoides (L.) L. }\end{array}$} & Whole plant & Antiparasitic & $\begin{array}{l}\text { Organic (hexane, } \\
\text { ethyl acetate, } \\
\text { chloroform, } \\
\text { methanol) and } \\
\text { aqueous extracts }\end{array}$ & $\begin{array}{l}\text { Trypanosoma brucei } \\
\text { Trypanosoma brucei } \\
\text { rhodesiense } \\
\text { Trypanosoma cruzi } \\
\text { Leishmania donovani } \\
\text { Plasmodium falciparum }\end{array}$ & In vitro & {$[98]$} \\
\hline & Whole plant & Chagas disease & $\begin{array}{l}\text { Aqueous and } \\
\text { ethanolic }\end{array}$ & Trypanosoma cruzi & In vitro & {$[149]$} \\
\hline & Whole plant & Antileishmanial & $\begin{array}{l}\text { Aqueous and } \\
\text { ethanolic }\end{array}$ & Leishmania amazonensis & In vitro & [149] \\
\hline & Leaf & Antiparasitic & $\begin{array}{l}\text { Aqueous and } \\
\text { ethanol extract }\end{array}$ & Heligmosomoides bakeri & In vitro & {$[157]$} \\
\hline & Leaf & Antiparasitic & Ethanol extract & Rhipicephalus microplus & In vitro & {$[115]$} \\
\hline \multirow{4}{*}{$\begin{array}{l}\text { Artemisia absinthium } \\
\mathrm{L} .\end{array}$} & Flower & Antiparasitic & Di-ethyl ether & Toxocara cati & In vivo & {$[163]$} \\
\hline & & & essential oil & $\begin{array}{l}\text { Trypanosoma cruzi } \\
\text { Trichomonas vaginalis }\end{array}$ & In vitro & {$[74]$} \\
\hline & & & & $\begin{array}{l}\text { Trypanosoma cruzi } \\
\text { Leishmania infantum }\end{array}$ & In vitro & {$[5]$} \\
\hline & Leaf & Schistosomicidal & Dichloromethane & Schistosoma mansoni & In vitro & {$[20]$} \\
\hline \multirow[t]{3}{*}{$\begin{array}{l}\text { Artemisia abyssinica } \\
\text { Sch. Bip. ex A. Rich. }\end{array}$} & Aerial part & Antitrypanosomal & $\begin{array}{l}\text { Dichloromethane: } \\
\text { Methanol }\end{array}$ & $\begin{array}{l}\text { Trypanosoma brucei } \\
\text { brucei }\end{array}$ & In vitro & {$[94]$} \\
\hline & & & Dichloromethane & $\begin{array}{l}\text { Trypanosoma } \\
\text { congolense }\end{array}$ & In vivo & {$[25]$} \\
\hline & Aerial part & Antitrypanosomal & $\begin{array}{l}\text { Dichloromethane: } \\
\text { Methanol }\end{array}$ & $\begin{array}{l}\text { Trypanosoma brucei } \\
\text { brucei }\end{array}$ & In vitro & {$[94]$} \\
\hline \multirow[t]{3}{*}{$\begin{array}{l}\text { Artemisia afra Jacq. } \\
\text { ex Willd. }\end{array}$} & Leaf & Antitrypanosomal & Dichloromethane & $\begin{array}{l}\text { Trypanosoma brucei } \\
\text { rhodesiense / } \\
\text { Trypanosoma cruzi. }\end{array}$ & In vitro & {$[82]$} \\
\hline & & Antitrypanosomal & $\begin{array}{l}\text { Dichloromethane: } \\
\text { methanol }\end{array}$ & $\begin{array}{l}\text { Trypanosoma brucei } \\
\text { brucei }\end{array}$ & In vitro & {$[94]$} \\
\hline & & Antimalarial & Acetone & $\begin{array}{l}\text { Plasmodium falciparum } \\
\text { NF54 }\end{array}$ & In vitro & {$[85]$} \\
\hline Artemisia annua L. & Aerial part & Antitrypanosomal & $\begin{array}{l}\text { Dichloromethane: } \\
\text { Methanol }\end{array}$ & $\begin{array}{l}\text { Trypanosoma brucei } \\
\text { brucei }\end{array}$ & In vitro & {$[94]$} \\
\hline $\begin{array}{l}\text { Artemisia } \\
\text { herba-alba Asso }\end{array}$ & - & Antileishmanial & Aqueous & Leishmania tropica & In vitro & {$[43]$} \\
\hline $\begin{array}{l}\text { Baccharis salicifolia } \\
\text { (Ruiz \& Pav.) Pers. }\end{array}$ & Leaf & Antileishmanial & $\begin{array}{l}\text { Ethyl acetate } \\
\text { extract }\end{array}$ & Leishmania braziliensis & In vitro & {$[27]$} \\
\hline Baccharis uncinella DC. & Leaf & Antileishmanial & Ursolic acid & Leishmania infantum & In vivo & {$[49]$} \\
\hline
\end{tabular}


Table 2. (continued).

\begin{tabular}{|c|c|c|c|c|c|c|}
\hline Plant $^{1}$ & $\begin{array}{l}\text { Plant part } \\
\text { used }\end{array}$ & Pharmacological & Preparation & Organism tested & $\begin{array}{l}\text { Context } \\
\text { of use }\end{array}$ & Reference \\
\hline \multirow[t]{6}{*}{ Bidens pilosa $\mathrm{L}$. } & \multirow[t]{6}{*}{ Leaf } & Antimalarial & $\begin{array}{l}\text { Organic extracts } \\
\text { and fractions }\end{array}$ & Plasmodium falciparum & In vitro & {$[13]$} \\
\hline & & Antimalarial & Organic extracts & Plasmodium falciparum & In vitro & {$[102]$} \\
\hline & & Antimalarial & Organic extracts & Plasmodium falciparum & In vitro & {$[151]$} \\
\hline & & Antimalarial & Organic extracts & $\begin{array}{l}\text { Plasmodium falciparum, } \\
\text { Plasmodium berghei } \\
\text { NK-65 }\end{array}$ & $\begin{array}{l}\text { in vitro \& } \\
\text { in vivo } \\
\text { (mice) }\end{array}$ & {$[63]$} \\
\hline & & Anthelmintic & Ethanol extract & Haemonchus contortus & In vitro & {$[36]$} \\
\hline & & Antileishmanial & Crude extracts & Leishmania amazonensis & In vitro & {$[35,49,151]$} \\
\hline $\begin{array}{l}\text { Blumea lacera } \\
\text { (Burm.f.) DC. }\end{array}$ & Leaf & Anthelmintic & $\begin{array}{l}\text { Alcoholic and } \\
\text { aqueous extracts }\end{array}$ & $\begin{array}{l}\text { Ascaris lumbricoides } \\
\text { Pheretima posthuma }\end{array}$ & In vitro & {$[119]$} \\
\hline \multirow{2}{*}{$\begin{array}{l}\text { Calendula } \\
\text { officinalis L. }\end{array}$} & \multirow[t]{2}{*}{ Flower } & Antileishmanial & Methanol (80\%) & Leishmania major & In vitro & {$[95]$} \\
\hline & & Antiparasitic & $\begin{array}{l}\text { Oleanolic acid } \\
\text { and its glycosides }\end{array}$ & $\begin{array}{l}\text { Heligmosomoides } \\
\text { polygyrus }\end{array}$ & $\begin{array}{l}\text { in vitro \& } \\
\text { in vivo } \\
\text { (mice) }\end{array}$ & {$[145]$} \\
\hline $\begin{array}{l}\text { Centipeda } \\
\text { minima (L.) }\end{array}$ & \multirow[t]{3}{*}{ Whole plant } & \multirow[t]{3}{*}{ Antiparasitic } & \multirow[t]{3}{*}{$\begin{array}{l}\text { Crude extracts } \\
\text { and fractions }\end{array}$} & Giardia intestinalis & In vitro & {$[164]$} \\
\hline \multirow[t]{2}{*}{ A. Braun \& Asch. } & & & & Entamoeba histolytica & & \\
\hline & & & & Plasmodium falciparum & & \\
\hline \multirow[t]{2}{*}{$\begin{array}{l}\text { Chersodoma } \\
\text { jodopappa Cabrera }\end{array}$} & Leaf & Antileishmanial & Ethanol extract & $\begin{array}{l}\text { Leishmania amazonensis } \\
\text { Leishmania braziliensis }\end{array}$ & In vitro & {$[27]$} \\
\hline & Stem & Antileishmanial & Ethanol extract & Leishmania donovani & In vitro & {$[27]$} \\
\hline \multirow[t]{2}{*}{ Cichorium intybus L. } & \multirow[t]{2}{*}{ Leaf } & \multirow[t]{2}{*}{ Anthelmintic } & \multirow[t]{2}{*}{ Methanol:water } & Ascaris suum & In vitro & {$[160]$} \\
\hline & & & & $\begin{array}{l}\text { Oesophagostomum } \\
\text { dentatum }\end{array}$ & & \\
\hline \multirow{3}{*}{$\begin{array}{l}\text { Cnicothamnus } \\
\text { lorentzii Griseb. }\end{array}$} & Leaf & Antileishmanial & Ethanol extract & Leishmania amazonensis & In vitro & {$[27]$} \\
\hline & & & & Leishmania donovani & & \\
\hline & Stem & Antileishmanial & Ethanol extract & Leishmania braziliensis & In vitro & {$[27]$} \\
\hline $\begin{array}{l}\text { Conyza albida Willd. } \\
\text { ex Spreng. }\end{array}$ & Whole plant & Antitrypanosomal & $\begin{array}{l}\text { Dichloromethane: } \\
\text { methanol }\end{array}$ & $\begin{array}{l}\text { Trypanosoma brucei } \\
\text { rhodesiense, } \\
\text { Trypanosoma cruzi }\end{array}$ & In vitro & {$[82]$} \\
\hline $\begin{array}{l}\text { Conyza podocephala } \\
\text { DC. }\end{array}$ & Whole plant & Antitrypanosomal & $\begin{array}{l}\text { Dichloromethane: } \\
\text { methanol }\end{array}$ & $\begin{array}{l}\text { Trypanosoma brucei } \\
\text { rhodesiense, } \\
\text { Trypanosoma cruzi }\end{array}$ & In vitro & {$[82]$} \\
\hline Conyza scabrida DC. & Leaf & Antitrypanosomal & $\begin{array}{l}\text { Dichloromethane: } \\
\text { methanol }\end{array}$ & $\begin{array}{l}\text { Trypanosoma brucei } \\
\text { rhodesiense, } \\
\text { Trypanosoma cruzi }\end{array}$ & In vitro & {$[82]$} \\
\hline $\begin{array}{l}\text { Echinacea purpurea } \\
\text { (L.) Moench }\end{array}$ & Whole part & Antileishmanial & Ethanol extract & Leishmania sp. & In vitro & {$[114]$} \\
\hline \multirow{2}{*}{$\begin{array}{l}\text { Eclipta alba (L.) } \\
\text { Hassk. }\end{array}$} & \multirow[t]{2}{*}{ Leaf } & Antimalarial & Crude extract & Plasmodium berghei & In vivo & {$[6]$} \\
\hline & & Antileishmanial & Crude extract & Leishmania donovani & In vitro & {$[138]$} \\
\hline $\begin{array}{l}\text { Eclipta prostrata } \\
\text { (L.) L. }\end{array}$ & Leaf & $\begin{array}{l}\text { Anthelmintic } \\
\text { activity }\end{array}$ & $\begin{array}{l}\text { Ethanol and } \\
\text { aqueous extracts }\end{array}$ & Pheretima posthuma & In vitro & {$[11]$} \\
\hline
\end{tabular}


Table 2. (continued).

\begin{tabular}{|c|c|c|c|c|c|c|}
\hline Plant $^{1}$ & $\begin{array}{l}\text { Plant part } \\
\text { used }\end{array}$ & Pharmacological & Preparation & Organism tested & $\begin{array}{l}\text { Context } \\
\text { of use }\end{array}$ & Reference \\
\hline & Leaf & $\begin{array}{l}\text { Anthelmintic } \\
\text { activity }\end{array}$ & Organic extracts & Pheretima posthuma & In vitro & {$[50]$} \\
\hline & Leaf & Antileishmanial & $\begin{array}{l}\text { Saponin, } \\
\text { dasyscyphin C }\end{array}$ & $\begin{array}{l}\text { Leishmania major, } \\
\text { Leishmania aethiopica, } \\
\text { Leishmania tropica }\end{array}$ & In vitro & {$[56]$} \\
\hline & Whole plant & $\begin{array}{l}\text { Anthelmintic } \\
\text { activity }\end{array}$ & $\begin{array}{l}\text { Organic and } \\
\text { water extracts }\end{array}$ & Haemonchus contortus & In vitro & [139] \\
\hline $\begin{array}{l}\text { Elephantopus } \\
\text { scaber L. }\end{array}$ & Leaf & Antitrypanosomal & $\begin{array}{l}\text { Organic extracts } \\
\text { and sesquiterpene } \\
\text { lactone }\end{array}$ & $\begin{array}{l}\text { Trypanosoma brucei } \\
\text { rhodesiense }\end{array}$ & In vitro & {$[165]$} \\
\hline $\begin{array}{l}\text { Helichrysum } \\
\text { nudifolium (L.) Less. }\end{array}$ & Whole plant & Antitrypanosomal & $\begin{array}{l}\text { Dichloromethane: } \\
\text { methanol }\end{array}$ & $\begin{array}{l}\text { Trypanosoma brucei } \\
\text { rhodesiense, } \\
\text { Trypanosoma cruzi }\end{array}$ & In vitro & {$[82]$} \\
\hline Inula montana $\mathrm{L}$. & Aerial part & Antileishmanial & Methanol & Leishmania infantum & In vitro & [73] \\
\hline $\begin{array}{l}\text { Jasonia glutinosa (L.) } \\
\text { DC. }\end{array}$ & Aerial part & Antileishmanial & Acetone & Leishmania donovani & In vitro & {$[156]$} \\
\hline $\begin{array}{l}\text { Kleinia odora } \\
\text { (Forssk.) DC. }\end{array}$ & Whole plant & Antiparasitic & $\begin{array}{l}\text { Ursane, } \\
\text { triterpenes } \\
\text { of lupane }\end{array}$ & $\begin{array}{l}\text { Trypanosoma brucei } \\
\text { Trypanosoma cruzi } \\
\text { Leishmania infantum } \\
\text { Plasmodium falciparum }\end{array}$ & In vitro & [89] \\
\hline \multirow{2}{*}{$\begin{array}{l}\text { Munnozia fournetii } \\
\text { H. Rob. (unresolved } \\
\text { name) }\end{array}$} & Leaf & Antileishmanial & Ethanol extract & Leishmania amazonensis & In vitro & {$[27]$} \\
\hline & Stem & Antileishmanial & Ethanol extract & $\begin{array}{l}\text { Leishmania donovani } \\
\text { Leishmania braziliensis }\end{array}$ & In vitro & {$[27]$} \\
\hline $\begin{array}{l}\text { Neurolaena lobate (L.) } \\
\text { R.Br. ex Cass. }\end{array}$ & Leaf & Antileishmanial & Ethanol extract & $\begin{array}{l}\text { Leishmania mexicana } \\
\text { Leishmania braziliensis }\end{array}$ & In vitro & [9] \\
\hline $\begin{array}{l}\text { Oedera genistifolia } \\
\text { (L.) Anderb. \& K. } \\
\text { Bremer }\end{array}$ & Whole plant & Antitrypanosomal & $\begin{array}{l}\text { Dichloromethane: } \\
\text { methanol }\end{array}$ & $\begin{array}{l}\text { Trypanosoma brucei } \\
\text { rhodesiense, } \\
\text { Trypanosoma cruzi }\end{array}$ & In vitro & {$[82]$} \\
\hline $\begin{array}{l}\text { Ophryosporus } \\
\text { piquerioides (DC.) } \\
\text { Benth. ex Baker }\end{array}$ & Whole plant & Antileishmanial & Ethanol extract & $\begin{array}{l}\text { Leishmania } \\
\text { amazonensis } \\
\text { Leishmania braziliensis }\end{array}$ & In vitro & {$[27]$} \\
\hline Pentzia globosa Less. & Root & Antitrypanosomal & $\begin{array}{l}\text { Dichloromethane: } \\
\text { methanol }\end{array}$ & $\begin{array}{l}\text { Trypanosoma brucei } \\
\text { rhodesiense } \\
\text { /Trypanosoma cruzi }\end{array}$ & In vitro & {$[82]$} \\
\hline $\begin{array}{l}\text { Perezia multiflora } \\
\text { (Humb. \& Bonpl.) } \\
\text { Less. }\end{array}$ & Leaf & Antileishmanial & Ethanol extract & $\begin{array}{l}\text { Leishmania amazonensis } \\
\text { Leishmania braziliensis } \\
\text { Leishmania donovani }\end{array}$ & In vitro & {$[27]$} \\
\hline $\begin{array}{l}\text { Pterocaulon } \\
\text { alopecuroideum Chodat } \\
\text { (unresolved name) }\end{array}$ & Whole plant & Antileishmanial & Ethanol extract & $\begin{array}{l}\text { Leishmania amazonensis } \\
\text { Leishmania braziliensis } \\
\text { Leishmania donovani }\end{array}$ & In vitro & {$[27]$} \\
\hline
\end{tabular}


Table 2. (continued).

\begin{tabular}{|c|c|c|c|c|c|c|}
\hline Plant $^{1}$ & $\begin{array}{l}\text { Plant part } \\
\text { used }\end{array}$ & Pharmacological & Preparation & Organism tested & $\begin{array}{l}\text { Context } \\
\text { of use }\end{array}$ & Reference \\
\hline \multirow{3}{*}{$\begin{array}{l}\text { Senecio clivicolus } \\
\text { Wedd. }\end{array}$} & Leaf & Antileishmanial & Ethanol extract & Leishmania amazonensis & In vitro & {$[27]$} \\
\hline & & & & Leishmania donovani & & \\
\hline & Stem & Antileishmanial & Ethanol extract & Leishmania braziliensis & In vitro & {$[27]$} \\
\hline $\begin{array}{l}\text { Solanecio mannii } \\
\text { (Hook. F) C. Jeffrey }\end{array}$ & Leaf & Antitrypanosomal & $\begin{array}{l}\text { Dichloromethane: } \\
\text { methanol }\end{array}$ & $\begin{array}{l}\text { Trypanosoma brucei } \\
\text { brucei }\end{array}$ & In vitro & {$[94]$} \\
\hline \multirow[t]{2}{*}{$\begin{array}{l}\text { Sphaeranthus } \\
\text { indicus } \mathrm{L} .\end{array}$} & Whole plant & Anthelmintic & $\begin{array}{l}\text { Ethanolic and } \\
\text { aqueous extracts }\end{array}$ & $\begin{array}{l}\text { Pheretima posthuma, } \\
\text { Ascaridia galli }\end{array}$ & In vitro & {$[134]$} \\
\hline & Leaf & $\begin{array}{l}\text { Macrofilaricidal } \\
\text { activity }\end{array}$ & Methanolic & Setaria digitata & In vitro & {$[96]$} \\
\hline \multirow{3}{*}{$\begin{array}{l}\text { Stevia yaconensis } \\
\text { Hieron. }\end{array}$} & Whole plant & Antileishmanial & Ethanol extract & Leishmania amazonensis & In vitro & {$[27]$} \\
\hline & & & & Leishmania braziliensis & & \\
\hline & & & & Leishmania donovani & & \\
\hline \multirow[t]{2}{*}{ Tagetes erecta $\mathrm{L}$. } & Root & Antimalarial & $\begin{array}{l}\text { Organic and } \\
\text { aqueous extracts }\end{array}$ & Plasmodium falciparum & In vitro & {$[41]$} \\
\hline & Flower & Anthelmintic & Organic extracts & Pheretima posthuma & In vitro & {$[106]$} \\
\hline $\begin{array}{l}\text { Tithonia diversifolia } \\
\text { (Hemsl.) A. Gray }\end{array}$ & Leaf & Antitrypanosomal & $\begin{array}{l}\text { Dichloromethane: } \\
\text { methanol }\end{array}$ & $\begin{array}{l}\text { Trypanosoma brucei } \\
\text { brucei }\end{array}$ & In vivo & {$[103]$} \\
\hline \multirow[t]{3}{*}{$\begin{array}{l}\text { Tridax procumbens } \\
\text { (L.) L. }\end{array}$} & Whole plant & $\begin{array}{l}\text { Antileishmanial } \\
\text { property }\end{array}$ & $\begin{array}{l}\text { Organic extracts } \\
\text { and (3S)-16,17 } \\
\text { didehydrofalcarinol }\end{array}$ & Leishmania mexicana & In vitro & {$[75]$} \\
\hline & & & $\begin{array}{l}\text { Methanol extract } \\
\text { and in combination } \\
\text { with Allium sativum }\end{array}$ & Leishmania mexicana & In vivo & {$[33]$} \\
\hline & & & $\begin{array}{l}\text { Oxylipin, (3S)-16,17- } \\
\text { didehydrofalcarinol }\end{array}$ & Leishmania mexicana & In vitro & {$[75]$} \\
\hline \multirow{3}{*}{$\begin{array}{l}\text { Vernonia } \\
\text { anthelmintica (L.) } \\
\text { Willd. }\end{array}$} & Whole plant & Anthelmintic & $\begin{array}{l}\text { Aqueous and } \\
\text { methanolic extracts }\end{array}$ & Haemonchus contortus & $\begin{array}{l}\text { in vitro \& } \\
\text { in vivo }\end{array}$ & {$[45]$} \\
\hline & Seed & Anthelmintic & Ethanolic extract & Haemonchus contortus & In vitro & {$[44]$} \\
\hline & Seed & Anthelmintic & - & Haemonchus contortus & $\begin{array}{l}\text { In vivo } \\
\text { (buffaloes) }\end{array}$ & {$[93]$} \\
\hline $\begin{array}{l}\text { Vernonia auriculifera } \\
\text { Hiern }\end{array}$ & Root & Antitrypanosomal & Dichloromethane & $\begin{array}{l}\text { Trypanosoma brucei } \\
\text { rhodesiense }\end{array}$ & In vitro & {$[29]$} \\
\hline $\begin{array}{l}\text { Vernonia hirsute } \\
\text { (DC.) Sch. Bip. ex } \\
\text { Walp. }\end{array}$ & Whole plant & Antitrypanosomal & $\begin{array}{l}\text { Dichloromethane: } \\
\text { methanol }\end{array}$ & $\begin{array}{l}\text { Trypanosoma brucei } \\
\text { rhodesiense, } \\
\text { Trypanosoma cruzi }\end{array}$ & In vitro & {$[82]$} \\
\hline $\begin{array}{l}\text { Vernonia mespilifolia } \\
\text { Less. }\end{array}$ & Leaf & Antitrypanosomal & $\begin{array}{l}\text { Dichloromethane: } \\
\text { methanol }\end{array}$ & $\begin{array}{l}\text { Trypanosoma brucei } \\
\text { rhodesiense, } \\
\text { Trypanosoma cruzi }\end{array}$ & In vitro & {$[82]$} \\
\hline $\begin{array}{l}\text { Vernonia natalensis } \\
\text { Oliv. \& Hiern }\end{array}$ & Whole plant & Antitrypanosomal & $\begin{array}{l}\text { Dichloromethane: } \\
\text { methanol }\end{array}$ & $\begin{array}{l}\text { Trypanosoma brucei } \\
\text { rhodesiense, } \\
\text { Trypanosoma cruzi }\end{array}$ & In vitro & {$[82]$} \\
\hline $\begin{array}{l}\text { Vernonia oligocephala } \\
\text { Katt }\end{array}$ & Leaf & Antitrypanosomal & Dichloromethane & $\begin{array}{l}\text { Trypanosoma brucei } \\
\text { rhodesiense, } \\
\text { Trypanosoma cruzi }\end{array}$ & In vitro & {$[82]$} \\
\hline
\end{tabular}


Table 2. (continued).

\begin{tabular}{llllll}
\hline Plant $^{1}$ & $\begin{array}{l}\text { Plant part } \\
\text { used }\end{array}$ & Pharmacological & Preparation & Organism tested & $\begin{array}{l}\text { Context } \\
\text { of use }\end{array}$ \\
\hline $\begin{array}{llll}\text { Vernonia squamulose } \\
\text { Hook. \& Arn. }\end{array}$ & Stem & Antileishmanial & Ethanol extract & $\begin{array}{l}\text { Leishmania amazonensis } \\
\text { In vitro }\end{array}$ & {$[27]$} \\
& & & $\begin{array}{l}\text { Leishmania braziliensis } \\
\text { Leishmania donovani }\end{array}$ &
\end{tabular}

\begin{tabular}{|c|c|c|c|c|c|}
\hline \multirow[t]{2}{*}{$\begin{array}{l}\text { Werneria nubigena } \\
\text { Kunth }\end{array}$} & Leaf & Antileishmanial & Ethanol extract & $\begin{array}{l}\text { Leishmania amazonensis } \\
\text { Leishmania donovani }\end{array}$ & In vitro \\
\hline & Stem & Antileishmanial & Ethanol extract & Leishmania braziliensis & In vitro \\
\hline \multirow[t]{2}{*}{$\begin{array}{l}\text { Xanthium catharticum } \\
\text { Kunth }\end{array}$} & Leaf & Antileishmanial & Ethanol extract & $\begin{array}{l}\text { Leishmania amazonensis } \\
\text { Leishmania donovani }\end{array}$ & In vitro \\
\hline & Stem & Antileishmanial & Ethanol extract & Leishmania braziliensis & In vit \\
\hline \multirow[t]{2}{*}{$\begin{array}{l}\text { Xanthium strumarium } \\
\text { L. }\end{array}$} & Leaf & Antitrypanosomal & $\begin{array}{l}50 \% \text { ethanolic } \\
\text { extract }\end{array}$ & Trypanosoma evansi & $\begin{array}{l}\text { In vitro } \\
\text { and in }\end{array}$ \\
\hline & Fruit & Antimalarial & $\begin{array}{l}\text { Methanol: water } \\
\text { extract }\end{array}$ & $\begin{array}{l}\text { Plasmodium falciparum } \\
\text { strain FCR-3 }\end{array}$ & \\
\hline
\end{tabular}

$\overline{{ }^{1} \text { All taxonomic names were verified in the Global Composite Checklist database (http://compositae.landcareresearch.co.nz/Default.aspx) }}$

flavanone $>$ isoflavone $>$ glucorhamnosyl-flavone. The affinity was dependent on the presence of hydroxyl groups at positions C- 5 and C-3, and was further increased by a hydrophobic 1,1-dimethylallyl substituent at position C-8.

Brandio et al. [13] first reported the antimalarial activity of crude extracts and their fractions from different species of Bidens, and provided evidence that this is due to the presence of polyacetylene and flavonoids. Later, Kumari et al. [63] and Tobinaga et al. [151] isolated the polyacetylene compound (R)-1,2-dihydroxytrideca3,5,7,9,11-pentayne from leaf extracts of B. pilosa, which showed promising antimalarial activity against Plasmodium falciparum (Table 3). Moreover, this compound was tested in an in vivo model (mice infected with Plasmodium berghei NK-65 strain), and results showed that the compound can decrease the average parasitaemia in red blood cells, but further studies addressing its mechanism are required. The genus Calendula is very well studied for its phytochemistry, with triterpene alcohols, triterpene saponins, flavonoids, carotenoids and polysaccharides as the major classes of phytoconstituents. Szakie et al. [145] isolated several oleanolic acid glycoside derivatives and tested them against Heligmosomoides polygyrus; the wormicidal activity of the oleanolic acid glycosides was superior to that of the aglycone, and the level of activity was dependent on the nature of the sugar side-chain at the C-3 position. The first sugar molecule of the glucuronides, i.e., the glucuronic acid attached to the aglycone, appeared to be vital for the antiparasitic properties of these compounds [145]. E. prostrata was studied by several scientists for its antiparasitic properties such as antimalarial [6], antileishmanial [56,138], and anthelmintic activities $[11,50]$. Khanna et al. [56] isolated dasyscyphin $\mathrm{C}$ from the leaves and proved its antileishmanial activities against Leishmania major, Leishmania aethiopica and
Leishmania tropica (Table 3). A sesquiterpene lactone (deoxyelephantopin) was isolated by Zahari et al. [165] from E. scaber and proved active against Trypanosoma brucei rhodesience. Similarly, T. procumbens showed significant antileishmanial activity against promastigotes of Leishmania mexicana. The active principle was found to be an oxylipin, namely (3S)-16, 17- didehydrofalcarinol [76].

\section{Antiparasitic activity of flavonoids and terpenoids documented in Asteraceae}

Flavonoids are the class of compound of highest occurrence, wide structural diversity, and chemical stability. They have been isolated on a large scale from Asteraceae species and can be used as taxonomic markers at lower hierarchical levels [75]. Flavones and flavonols are common throughout the Asteraceae, i.e., glycosides of apigenin, luteolin, kaempferol, quercetin, flavanone derivatives, (-)-epicatechin and (-)-epigallocatechin (Figure 1). Although there are fewer reports on antigiardial activity in Asteraceae, these compounds from other families are well-studied against $G$. lamblia. From the aerial parts of Helianthemum glomeratum (Cistaceae), kaempferol, quercetin, (-)-epicatechin and (-)-epigallocatechin have shown antigiardial activity against G. lamblia (in vitro), with $\mathrm{IC}_{50}$ values of $26.47,8.73$, 1.64 and $8.06 \mu \mathrm{g} / \mathrm{mL}$, respectively [17]. Structure-activity correlation implies that the 2,3-double bond and 4-keto group of flavones might not be required for antiprotozoal activity since both (-)-epicatechin and (-)-epigallocatechin lack these structural units, yet maintain biological activity (Figure 1). Also, unlike flavones, the benzenediol moiety of $(-)$-epicatechin and $(-)$ epigallocatechin is not coplanar with the heterocyclic part because C-2 of their 
Table 3. List of compounds from Asteraceae commonly reported for their antiparasitic properties.

\begin{tabular}{llll}
\hline Plant $^{1}$ & Name of the compounds/group & Organism tested & References \\
\hline Acanthospermum hispidum DC. & Sesquiterpenic lactones & Plasmodium falciparum & [34]
\end{tabular}

Acmella ciliate (Kunth) Cass. Spilanthol

Ageratum conyzoides (L.) L. Methoxylated flavonoids

Ambrosia tenuifolia Spreng.

Ambrosia tenuifolia Spreng. and Ambrosia scabra Hook. \& Arn.

Artemisia annua L.

Aspilia africana (Pers.) C. D.

Adams

Baccharis retusa DC.

Baccharis uncinella DC.

Bidens pilosa L.

Bidens sulphurea (Cav.) Sch. Bip.

Calendula officinalis L.

Centipeda minima (L.)

A. Braun \& Asch.

Chromolaena odorata f. odorata

Cichorium intybus L.

Coreopsis lanceolate $\mathrm{L}$.

Dicoma tomentosa Cass.

Dicoma anomala subsp. gerrardii (Harv. ex F. C. Wilson) S. Ortiz \& Rodr. Oubiña

Eclipta prostrata (L.) L.
Psilostachyin

Peruvin

Psilostachyin and psilostachyin $\mathrm{C}$

Sesquiterpenes and sesquiterpene lactones

Thiarubrine A

Sakuranetin

Caffeic acid

Pectolinarigenin

Polyacetylene

2,6-Di-tert-butyl-4-methylphenol, germacrene $\mathrm{D}, \beta$-caryophyllene

Glycosides of oleanolic acid

Sesquiterpene lactone, brevilin A

Quercetin-4'-methyl ether

Sesquiterpene lactone

1-Phenylhepta-1,3,5-triyne and 5phenyl-2-(1'-propynyl)-thiophene

Sesquiterpene lactones

Eudesmanolide-type sesquiterpene lactone

Dasyscyphin C
Trypanosoma brucei rhodesiense and Plasmodium falciparum

Trypanosoma brucei rhodesiense, Trypanosoma cruzi, Leishmania donovani and Plasmodium falciparum

Leishmania mexicana

Trypanosoma cruzi

Plasmodium falciparum

Caenorhabditis elegans

Leishmania sp.

Leishmania amazonensis

Leishmania braziliensis

Plasmodium falciparum

Heligmosomoides polygyrus

Giardia intestinalis

Entamoeba histolytica

Plasmodium falciparum

Plasmodium falciparum

Haemonchus contortus

Bursaphelenchus xylophilus and Caenorhabditis elegans

Plasmodium falciparum

3D7 and $\mathrm{W} 2$

Plasmodium falciparum D10

Leishmania major, 
Table 3. (continued).

\begin{tabular}{|c|c|c|c|}
\hline Plant $^{1}$ & Name of the compounds/group & Organism tested & References \\
\hline Elephantopus scaber L. & Deoxyelephantopin & $\begin{array}{l}\text { Trypanosoma brucei rhodesience, } \\
\text { strain STIB } 900\end{array}$ & {$[165]$} \\
\hline Fructus arctii & Arctigenin and arctiin & Dactylogyrus intermedius & {$[158]$} \\
\hline Heterotheca inuloides Cass. & $\begin{array}{l}\text { 7-Hydroxy-3,4-dihydrocadalene, } \\
\text { 7-hydroxycalamenene }\end{array}$ & Giardia intestinalis & {$[129]$} \\
\hline Kleinia odora (Forssk.) DC. & Ursolic acid and derivatives & $\begin{array}{l}\text { Plasmodium falciparum } \\
\text { Leishmania infantum } \\
\text { Trypanosoma cruzi } \\
\text { Trypanosoma brucei }\end{array}$ & {$[89]$} \\
\hline Pentacalia desiderabilis Cuatrec. & Jacaranone & $\begin{array}{l}\text { Leishmania braziliensis } \\
\text { Leishmania amazonensis }\end{array}$ & {$[83]$} \\
\hline $\begin{array}{l}\text { Porophyllum ruderale (Jacq.) } \\
\text { Cass. }\end{array}$ & Thiophene derivatives & Leishmania amazonensis & {$[146]$} \\
\hline Sphaeranthus indicus L. & $\begin{array}{l}\text { Indicusalactone, }(-) \text {-oxyfrullanolide, } \\
\text { 7-Hydroxyfrullanolide, squalene, } \\
\text { 3,5-di-O-caffeoylquinic acid methyl } \\
\text { ester, 3,4-di-O-caffeoylquinic acid } \\
\text { methyl ester }\end{array}$ & Plasmodium falciparum & {$[132]$} \\
\hline Tagetes erecta $L$. & $\begin{array}{l}\text { 2-Hydroxymethyl-non-3-ynoic acid, } \\
\text { 2-[2,2']-bithiophenyl-5- ethyl ester }\end{array}$ & $\begin{array}{l}\text { Plasmodium falciparum MRC-pf-2 } \\
\text { Plasmodium falciparum MRC-pf-56 }\end{array}$ & {$[41]$} \\
\hline $\begin{array}{l}\text { Tagetes patula L. Synonym of } \\
\text { Tagetes erecta L. }\end{array}$ & $\alpha$-terthienyl, gallic and linoleic acids & Heterodera zeae & {$[24]$} \\
\hline Tridax procumbens (L.) L. & $\begin{array}{l}\text { (3s)-16,17-Didehydrofalcarinol, } \\
\text { (3S)-16,17-didehydrofalcarinol }\end{array}$ & $\begin{array}{l}\text { Leishmania mexicana } \\
\text { Leishmania mexicana }\end{array}$ & $\begin{array}{l}{[75]} \\
{[75]}\end{array}$ \\
\hline $\begin{array}{l}\text { Tanacetum parthenium (L.) Sch. } \\
\text { Bip. }\end{array}$ & Parthenolide & Leishmania amazonensis & {$[150]$} \\
\hline $\begin{array}{l}\text { Tithonia diversifolia (Hemsl.) A. } \\
\text { Gray }\end{array}$ & $\begin{array}{l}\text { Sesquiterpenes and sesquiterpene } \\
\text { lactones }\end{array}$ & Plasmodium falciparum & {$[38]$} \\
\hline $\begin{array}{l}\text { Trixis antimenorrhoea (Schrank) } \\
\text { Mart. ex Baker }\end{array}$ & Trixanolide & $\begin{array}{l}\text { Leishmania amazonensis } \\
\text { Leishmania braziliensis }\end{array}$ & {$[72]$} \\
\hline Vernonia amygdalina Delile & $\begin{array}{l}\text { Sesquiterpenes and sesquiterpene } \\
\text { lactones }\end{array}$ & Plasmodium falciparum & {$[100]$} \\
\hline Vernonia brachycalyx O. Hoffm. & Sesquiterpene dilactone & $\begin{array}{l}\text { Plasmodium falciparum }(\mathrm{K} 39,3 \mathrm{D} 7 \text {, } \\
\mathrm{V} 1 / \mathrm{S} \text { and } \mathrm{Dd} 2)\end{array}$ & [101] \\
\hline Vernonia angulifolia DC. & $\begin{array}{l}\text { Sesquiterpenes and sesquiterpene } \\
\text { lactones }\end{array}$ & Plasmodium falciparum & {$[121]$} \\
\hline Xanthium macrocarpum DC. & $\begin{array}{l}\text { Xanthanolides (xanthinosin } \\
\text { xanthatin, xanthinin, } \\
\text { 4-epiisoxanthanol, } \\
\text { 4-epixanthanol) }\end{array}$ & $\begin{array}{l}\text { Leishmania mexicana } \\
\text { Leishmania infantum }\end{array}$ & {$[65]$} \\
\hline
\end{tabular}

\footnotetext{
${ }^{1}$ All taxonomic names were verified in the Global Composite Checklist database (http://compositae.landcareresearch.co.nz/Default.aspx)
} 
<smiles>O=c1c(O)c(-c2ccc(O)c(O)c2)oc2cc(O)cc(O)c12</smiles><smiles>Oc1cc(O)c2c(c1)O[C@H](c1ccc(O)c(O)c1)[C@H](O)C2</smiles>

(a) quercetin<smiles>O=c1c(O)c(-c2ccc(O)cc2)oc2cc(O)cc(O)c12</smiles>

(d) Kaempferol (b) (-)-epicatechin<smiles>O=c1cc(-c2ccc(O)c(O)c2)oc2cc(O)cc(O)c12</smiles>

(e) Luteolin<smiles>Oc1cc(O)c2c(c1)O[C@H](c1cc(O)c(O)c(O)c1)[C@H](O)C2</smiles>

(c) (-) epigallocatechin<smiles>O=c1c(O)c(-c2ccc(O)c(O)c2)oc2cc(O)ccc12</smiles>

(f) Fisetin

Figure 1. Common flavonoids of the Asteraceae family reported as antiparasitic compounds

flavan-3-ol structure is an sp3 carbon. In addition, there are several reports that glycosylated flavonoids also possess antigiardial activity. Also, a C-3 glycosylated flavone tiliroside $[17,79]$, obtained from $H$. glomeratum, has been shown to possess antigiardial inhibitory activity with an $\mathrm{IC}_{50}$ value of $17.36 \mu \mathrm{g} / \mathrm{mL}$.

Recently, Klongsiriwet et al. [57] demonstrated that quercetin and luteolin are highly effective at $250 \mu \mathrm{M}$ to reduce the in vitro exsheathment of Haemonchus contortus L3 larvae. Tasdemir et al. studied the antitrypanosomal and antileishmanial activities of flavonoids and their analogues in vitro and in vivo, as well as their (quantitative) structure-activity relationship [148]. They showed that fisetin, 3-hydroxyflavone, luteolin, and quercetin are the most potent antileishmanial compounds against Leishmania donovani, with $\mathrm{IC}_{50}$ of $0.6,0.7,0.8$, and $1.0 \mu \mathrm{g} / \mathrm{mL}$, respectively (Table 4). Moreover, these authors found moderate antitrypanosomal efficacy of these compounds against Trypanosoma brucei rhodesiense and Trypanosoma cruzi. The authors conclude that 7,8-dihydroxyflavone and quercetin appeared to ameliorate parasitic infections in mouse models, and are potent and effective antiprotozoal agents. Mead and McNair [78] also studied the antiparasitic activity of flavonoids and isoflavones against Cryptosporidium parvum and Encephalitozoon intestinalis. These authors also found that quercetin and apigenin had activity against Encephalitozoon intestinalis at $\mathrm{EC}_{50}$ of 15 and $50 \mathrm{mM}$, respectively, while low activity of luteolin and quercetin was found against Cryptosporidium parvum. No inhibition was observed with either rutin or epigallocatechin gallate against either parasite. Lehane and Saliba [66] investigated the effects of a range of common dietary flavonoids on the growth of two strains of the human malaria parasite Plasmodium falciparum and concluded that luteolin showed $\mathrm{IC}_{50}$ values of $11 \pm 1 \mu \mathrm{M}$ and $12 \pm 1 \mu \mathrm{M}$ for strains 3D7 and 7G8, respectively. Although luteolin was found to prevent the progression of parasite growth beyond the young trophozoite stage, it did not affect parasite susceptibility to the antimalarial drugs chloroquine or artemisinin. Nour et al., [98] found moderate antiparasitic activity of five methoxylated flavonoids viz. 5,6,7,8,5pentamethoxy-3,4-methylenedioxyflavone (eupalestin), 5,6,7,5-tetramethoxy-3,4-methylenedioxyflavone; 5,6,7,8, 3,4,5-heptamethoxy-flavone (5-methoxynobiletine), 5,6, 7,3,4,5-hexamethoxy-flavone and 4-hydroxy-5,6,7,3,5-pentamethoxy-flavone (ageconyflavone) against several parasites: Trypanosoma brucei rhodesiense, Trypanosoma cruzi, Leishmania donovani and Plasmodium falciparum (Table 4).

Terpenoids are the largest group of phytochemicals as they comprise more than 20,000 recognised molecules. Depending on the number of carbons, terpenoids are divided into classes, starting with sesquiterpenes and continuing with diterpenes, sterols, triterpenes and finally tetraterpenes. Several sesquiterpenes, sterols and triterpenes have been isolated from members of the Asteraceae family. The sesquiterpenes commonly found in leaf extracts from Asteraceae are divided into mono- and bicyclic. The most abundant sterols from Asteraceae are stigmasterol and sitosterol. Sequiterpenes isolated from Vernonia spp. have antiparasitic activity against Plasmodium falciparum. Four compounds such as vernodalin, vernodalol, vernolide, and hydroxyvernolide (Figure 2), all derived from the leaves of Vernonia amygdalina, have potent activity with $\mathrm{IC}_{50}$ values of $4,4.2,8.4$ and $11.4 \mu \mathrm{g} /$ $\mathrm{mL}$, respectively [60]. Another compound: sesquiterpene dilactone (16,17-dihydrobrachycalyxolide), isolated from the leaves of $V$. brachycalyx, exhibited anti-plasmodial activity against different multidrug-resistant strains of 
Table 4. Selected flavonoids and terpenoids (whose presence has been reported in plants of the Asteraceae family) with antiparasitic activity

\begin{tabular}{|c|c|c|c|}
\hline Flavonoids & Organism tested & $\begin{array}{l}\text { Concentration/ } \\
\text { dose } \mathrm{IC}_{50}\end{array}$ & References \\
\hline Four polyoxygenated flavonoids & Trypanosoma brucei rhodesiense & $\begin{array}{l}\mathrm{C} 1: 16 \mu \mathrm{M}, \mathrm{C} 2: 18 \mu \mathrm{M}, \\
\mathrm{C} 3: 21 \mu \mathrm{M} \text { and } \mathrm{C} 4: \\
11 \mu \mathrm{M}\end{array}$ & [97] \\
\hline
\end{tabular}

5,6,7,8,5-Pentamethoxy-3,4-methylenedioxy flavone
Trypanosoma brucei rhodesiense;

Trypanosoma cruzi;

Leishmania donovani and

Plasmodium falciparum

5,6,7,5-Tetramethoxy-3,4-methylenedioxyflavone Trypanosoma brucei rhodesiense; Trypanosoma cruzi;

Leishmania donovani and

Plasmodium falciparum

$5,6,7,8,3,4,5$-Hepta-methoxyflavone

5,6,7,3,4,5-Hexamethoxyflavone

4-Hydroxy-5,6,7,3,5-pentamethoxyflavone (ageconyflavone $\mathrm{C}$ )

3, 5, 7, 3'-Tetrahydroxy-4'-methoxyflavone

Bractein

Kaempferol

Quercetin

(-)-Epicatechin

(-)-Epigallocatechin

Quercetin

Luteolin

Quercetin

Fisetin
Trypanosoma brucei rhodesiense;

Trypanosoma cruzi;

Leishmania donovani and

Plasmodium falciparum

Trypanosoma brucei rhodesiense;

Trypanosoma cruzi;

Leishmania donovani and

Plasmodium falciparum

Trypanosoma brucei rhodesiense;

Trypanosoma cruzi;

Leishmania donovani and

Plasmodium falciparum

Plasmodium falciparum

Leishmania donovani

Giardia lamblia

Giardia lamblia

Giardia lamblia

Giardia lamblia

Haemonchus contortus

Haemonchus contortus

Leishmania donovani

Leishmania donovani

Leishmania donovani
Tb: $6.67 \mu \mathrm{g} / \mathrm{mL}$

Tc- $>30 \mu \mathrm{g} / \mathrm{mL}$ Ld: $>30 \mu \mathrm{g} / \mathrm{mL}$ Pf: $4.57 \mu \mathrm{g} / \mathrm{mL}$

Tb: $7.29 \mu \mathrm{g} / \mathrm{mL}$ Tc: $19.5 \mu \mathrm{g} / \mathrm{mL}$ Ld: $>30 \mu \mathrm{g} / \mathrm{mL}$ Pf: $4.26 \mu \mathrm{g} / \mathrm{mL}$

Tb: $4.76 \mu \mathrm{g} / \mathrm{mL}$ Tc; $26.4 \mu \mathrm{g} / \mathrm{mL}$ Ld: $5.29 \mu \mathrm{g} / \mathrm{mL}$ Pf: $>5 \mu \mathrm{g} / \mathrm{mL}$

Tb: $8.58 \mu \mathrm{g} / \mathrm{mL}$ Tc: $>30 \mu \mathrm{g} / \mathrm{mL}$ $\mathrm{Ld}: 8.61 \mu \mathrm{g} / \mathrm{mL}$ Pf: $2.99 \mu \mathrm{g} / \mathrm{mL}$

Tb: $3.01 \mu \mathrm{g} / \mathrm{mL}$

Tc: $>30 \mu \mathrm{g} / \mathrm{mL}$ Ld: $3.56 \mu \mathrm{g} / \mathrm{mL}$ Pf: $3.59 \mu \mathrm{g} / \mathrm{mL}$

$26.47 \mu \mathrm{g} / \mathrm{mL}$

$8.73 \mu \mathrm{g} / \mathrm{mL}$

$1.64 \mu \mathrm{g} / \mathrm{mL}$

$8.06 \mu \mathrm{g} / \mathrm{mL}$

$250 \mu \mathrm{g} / \mathrm{mL}$ as highest concentration

$250 \mu \mathrm{g} / \mathrm{mL}$ as highest concentration

$0.8 \mu \mathrm{g} / \mathrm{mL}$

$1 \mu \mathrm{g} / \mathrm{mL}$

$0.6 \mu \mathrm{g} / \mathrm{mL}$ 
Table 4. (continued).

\begin{tabular}{|c|c|c|c|}
\hline Flavonoids & Organism tested & $\begin{array}{l}\text { Concentration/ } \\
{\text { dose } \mathrm{IC}_{50}}\end{array}$ & References \\
\hline 3-Hydroxyflavone & Leishmania donovani & $0.7 \mu \mathrm{g} / \mathrm{mL}$ & [148] \\
\hline Luteolin & Plasmodium falciparum 3D7 and 7G8 & $\begin{array}{l}\text { 3D7: } 11 \mu \mathrm{g} / \mathrm{mL} \\
\text { 7G8: } 12 \mu \mathrm{g} / \mathrm{mL}\end{array}$ & [66] \\
\hline
\end{tabular}

Terpenoids

Vernodalin

Vernodalol

Vernolide

Hydroxyvernolide

16,17- Dihydrobrachycalyxolide

Tagitinin C

15-Acetoxy-8 $\beta$-[(2-methylbutyryloxy)]-14-oxo-4, Plasmodium falciparum 3D7

5 -cis-acanthospermolide)

$9 \alpha$-Acetoxy-15-hydroxy- $8 \beta$ - $(2-$

methylbutyryloxy)-14-oxo- 4,5 -Trans-

acanthospermolide

$3 \beta$-Hydroxyolean-12-en-28-oic acid (oleanolic acid)

$3 \beta$-Hydroxyurs-12-en-28-oic acid (ursolic acid)

Indicusalactone

(-)-Oxyfrullanolide

7-Hydroxyfrullanolide,

Squalene

3,5-Di-O-caffeoylquinic acid methyl ester

(3s)-16,17-Didehydrofalcarinol
Leishmania amazonensis

Leishmania braziliensis

Plasmodium falciparum

Plasmodium falciparum

Plasmodium falciparum

Plasmodium falciparum

Plasmodium falciparum (K39, 3D7, $\mathrm{V} 1 / \mathrm{S}$ and Dd2)

Plasmodium falciparum 3D7

Leishmania infantum

Trypanosoma brucei

Trypanosoma cruzi

Plasmodium falciparum

Plasmodium falciparum

Plasmodium falciparum

Plasmodium falciparum

Plasmodium falciparum

Plasmodium falciparum

Leishmania mexicana
$4 \mu \mathrm{g} / \mathrm{mL}$

$4.2 \mu \mathrm{g} / \mathrm{mL}$

$8.4 \mu \mathrm{g} / \mathrm{mL}$

$11.4 \mu \mathrm{g} / \mathrm{mL}$

K39: $4.2 \mu \mathrm{g} / \mathrm{mL}$

3D7: $13.7 \mu \mathrm{g} / \mathrm{mL}$

V1/S: $3 \mu \mathrm{g} / \mathrm{mL}$

$\operatorname{Dd} 2: 16 \mu \mathrm{g} / \mathrm{mL}$

$0.75 \mu \mathrm{g} / \mathrm{mL}$

$2.9 \mu \mathrm{g} / \mathrm{mL}$

$2.23 \mu \mathrm{g} / \mathrm{mL}$

La: $>100 \mu \mathrm{g} / \mathrm{mL}$

[116,162], [161]

Li: $7.4 \mu \mathrm{M}$

Tb: $2.2 \mu \mathrm{M}$

Tc: $8.8 \mu \mathrm{M}$

Pf: $29.7 \mu \mathrm{M}$

$2.8 \mu \mathrm{g} / \mathrm{mL}$

$3.8 \mu \mathrm{g} / \mathrm{mL}$

$2.5 \mu \mathrm{g} / \mathrm{mL}$

$2.3 \mu \mathrm{g} / \mathrm{mL}$

$2.4 \mu \mathrm{g} / \mathrm{mL}$

$0.48 \mu \mathrm{M}$ 
Table 4. (continued).

\begin{tabular}{|c|c|c|c|}
\hline Flavonoids & Organism tested & $\begin{array}{l}\text { Concentration/ } \\
\text { dose } \mathrm{IC}_{50}\end{array}$ & References \\
\hline \multirow[t]{2}{*}{ Ursolic acid } & Leishmania amazonensis & $6.4 \mu \mathrm{g} / \mathrm{mL}$ & {$[162]$} \\
\hline & Leishmania infantum & $\begin{array}{l}\text { In vivo } 1.0 \mathrm{mg} / \mathrm{kg} \text { body } \\
\text { weight (mice) }\end{array}$ & [49] \\
\hline \multirow[t]{4}{*}{ Urs-12-ene- $3 \beta, 16 \beta$-diol } & Plasmodium falciparum & Pf: $9.7 \mu \mathrm{M}$ & [89] \\
\hline & Leishmania infantum & Li: $9.3 \mu \mathrm{M}$ & \\
\hline & Trypanosoma cruzi & Tc: $9.9 \mu \mathrm{M}$ & \\
\hline & Trypanosoma brucei & Tb: $2.3 \mu \mathrm{M}$ & \\
\hline \multirow[t]{4}{*}{$3 \beta, 11 \alpha$-Dihydroxyurs-12-ene } & Plasmodium falciparum & Pf: $23.9 \mu \mathrm{M}$ & [89] \\
\hline & Leishmania infantum & Li: $3.2 \mu \mathrm{M}$ & \\
\hline & Trypanosoma cruzi & Tc: $8.1 \mu \mathrm{M}$ & \\
\hline & Trypanosoma brucei & $\mathrm{Tb}: 7.8 \mu \mathrm{M}$ & \\
\hline \multirow[t]{2}{*}{ Betulinic acid } & Caenorhabditis elegans & $100 \mu \mathrm{g} / \mathrm{mL}$ & {$[22]$} \\
\hline & Plasmodium falciparum $\mathrm{W} 2$ & $2.33 \mu \mathrm{g} / \mathrm{mL}$ & {$[91]$} \\
\hline$\beta$-Sitosterol & Trypanosoma brucei brucei S427 & $12.5 \mu \mathrm{g} / \mathrm{mL}$ & {$[99]$} \\
\hline
\end{tabular}

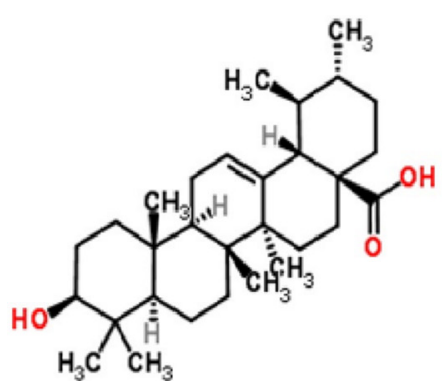

(g) ursolic acid<smiles>C=C[C@]12COC(=O)C(=C)[C@]1(C)[C@H]1OC(=O)C(=C)[C@H]1[C@@H]2OC(=O)C(=C)CO</smiles>

(j) Vernodalin

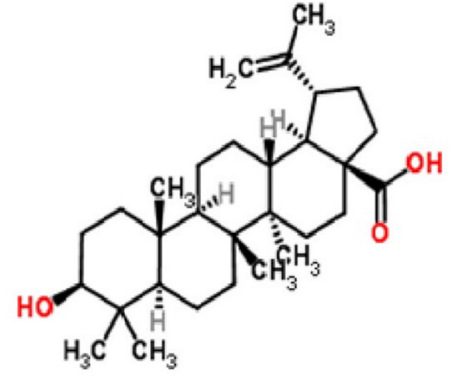

(h) betulinic acid<smiles>C=C[C@@]12C(=C)C(=O)OC[C@]1(C=C)[C@H](O)[C@H](C(=C)C(=O)OC)[C@@H]2OC(=O)C(=C)CO</smiles>

(k) Vernodalol<smiles>CCC(CC[C@@H](C)[C@H]1CC[C@H]2CC=C3C[C@H](O)CC[C@]3(C)[C@@H]2CC1)C(C)C</smiles>

(i) $\beta$-sitosterol

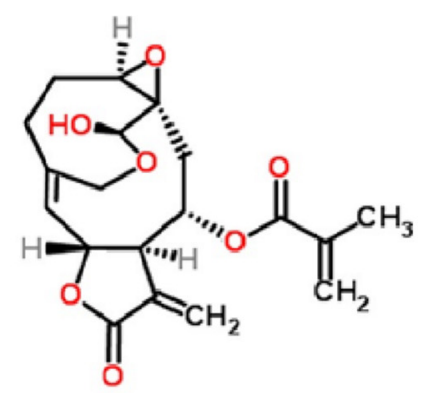

(1) Vernolide

Figure 2. Common terpenoids of the Asteraceae family reported as antiparasitic compounds

Plasmodium falciparum (K39, 3D7, V1/S and Dd2) with $\mathrm{IC}_{50}$ values of $4.2,13.7,3.0$, and $16 \mu \mathrm{g} / \mathrm{mL}$, respectively [101]. Goffin et al. [38] isolated the sesquiterpene lactone: tagitinin C, from the ether extract of Tithonia diversifolia and demonstrated antiplasmodial activity against Plasmodium falciparum $\left(\mathrm{IC}_{50}\right.$ of $\left.0.75 \mu \mathrm{g} / \mathrm{mL}\right)$. Becker et al. [8] identified urospermal A-15-O-acetate and dehydrobrachylaenolide as the main active compound responsible for the antiplasmodial activity against Plasmodium falciparum 3D7 and W2 strains. Ganfon et al. [34] investigated the antiparasitic activities of Acanthospermum hispidum by isolating two sesquiterpene lactones (15-acetoxy- $8 \beta$ [(2-methylbutyryloxy)]-14-oxo-4,5-cis-acanthospermolide), and $9 \alpha$-acetoxy-15-hydroxy- $8 \beta$-(2-methylbutyry499 loxy)-14-oxo-4,5-transacanthospermolide), both of which exhibited in vitro antiplasmodial activity against 
a chloroquine-sensitive strain (3D7) with $\mathrm{IC}_{50}$ values of 2.9 and $2.23 \mu \mathrm{M}$, respectively (Table 4 ).

Among the triterpenes, squalene and lupeol derivatives are the more common ones [67]. Oleanolic acid (3 $\beta$ hydroxyolean-12-en-28-oic acid) is a pentacyclic triterpenoid with widespread occurrence in Asteraceae and was found to have antimalarial and antileishmanial activity [89,162]. Recently, Yamamoto et al. [162] studied the activity of ursolic acid on Leishmania amazonensis (in vitro and in vivo). They found that ursolic acid eliminated Leishmania amazonensis promastigotes with an $\mathrm{EC}_{50}$ of $6.4 \mu \mathrm{g} / \mathrm{mL}$, comparable with miltefosine, while oleanolic acid presented only a marginal effect on promastigote forms at $100 \mu \mathrm{g} / \mathrm{mL}$. The possible mechanism by which promastigotes were eliminated by ursolic acid was programmed cell death, independent of caspase $3 / 7$, but it was highly dependent on mitochondrial activity. Also, the ursolic acid was not toxic for peritoneal macrophages from BALB/c mice, and it could eliminate intracellular amastigotes, associated with nitric oxide (NO) production. These authors conclude that ursolic acid can be considered an interesting candidate for future testing as a prototype drug for the treatment of cutaneous leishmaniasis. Enwerem et al. [22] examined the anthelmintic activity of betulinic acid on $C$. elegans and confirmed its strong anthelmintic activity at $100 \mu \mathrm{g} / \mathrm{mL}$, comparable to piperazine. Bringmann et al. [14] observed that betulinic acid exhibited moderate to good in vitro antimalarial activity against asexual erythrocytic stages of Plasmodium falciparum. Later, Steele et al. [141] concluded that betulinic acid can inhibit Plasmodium falciparum (in vitro), while in vivo experiments failed to reduce parasitaemia (up to $500 \mathrm{mg} / \mathrm{mL}$ in a murine malaria modelmice infected with $P$. berghei) and exhibited some toxicity. However, Ndjakou Lenta et al. [91] isolated betulinic acid, studied its in vitro activity against the Plasmodium falciparum W2 strain, and found it to be very potent with an $\mathrm{IC}_{50}$ of $2.33 \mu \mathrm{g} / \mathrm{mL}$. Nweze et al. [99] observed that $\beta$-sitosterol has modest anti-trypanosomal activity against Trypanosoma brucei $\mathrm{S} 427$ (in vitro $\mathrm{IC}_{50} 12.5 \mu \mathrm{g} / \mathrm{mL}$ ).

\section{Discussion}

In a review on nature-derived drugs, Zhu et al. [166] analysed "the ranking of drug-productive plant families based on the ratio of the approved drugs to reported bioactive natural products (including leads of the approved and clinical trials drugs)" and concluded that there are a few top-ranked plant families that produce high numbers of approved drugs among plant-derived medicines. According to Zhu et al. [166], Asteraceae is the fourth-largest drug-productive family that has yielded many approved drugs, including antiparasitic, anticancer, antiglaucoma, ant-inflammatory, antihepatotoxic, antiviral and choleretic agents. From 7229 Asteraceae species, 25 clinical drugs (17 approved and 8 in clinical trials) were documented among 1016 searchable drugs [91,99]. There are many FDA-approved nature-derived drugs that originate from Asteraceae as antiparasitics: arteether, artemether, artemisinin, artesunate, coarsucam, co-artemether, dihydroartemisinin and santonin (all from Artemisia species). Also, there are a few drugs still in clinical trials as antiparasitics, such as artemisone, arterolane and artelinic acid [92].

Traditional knowledge has proven a useful tool in the search for new plant-based medicines [18]. It has been estimated that the number of traditionally used plant species worldwide is between 10,000 and 53,000 [77]. In India alone, there are about 25,000 plant-based formulations used in folk and traditional medicine [126]. However, only a small proportion have been screened for biological activity $[42,140]$. Also, there are many specific regions that are less studied than others (only $1 \%$ of tropical floras have been investigated) [42]. Odisha's unique location in Peninsular India has blessed it with an interesting assemblage of floral and faunal diversity (http://odi shasbb.nic.in/index.php?lang=en). The state is on the eastern seaboard of India, located between $17^{\circ} 49^{\prime}$ and $22^{\circ}$ $36^{\prime} \mathrm{N}$ latitudes and between $81^{\circ} 36^{\prime}$ and $8^{\circ} 7 \quad 18^{\prime} \mathrm{E}$ longitudes. It covers an area of $1,55,707 \mathrm{sq} \mathrm{km}$ and is broadly divided into four geographical regions, i.e. the Northern Plateau (Chhotanagpur), Central River Basins, Eastern Hills and Coastal Plains. The confluence of two major biogeographic provinces of India: the Eastern Ghats (South-West) and Chhotanagpur Plateau (North), make Odisha a rich biodiversity repository with two internationally well-recognised areas: the Similipal Biosphere Reserve and the Chilika Lagoon. The state has a biodiversity board (it is a statutory body established under the Biological Diversity Act of 2002), with a network of 19 wildlife sanctuaries, one national park, one proposed national park, one biosphere reserve, two tiger reserves and three elephant reserves (http://odishasbb. nic.in/index.php?lang=en). Throughout the state, one finds varied and widespread forests harbouring different types of vegetation such as semi-evergreen forests, tropical moist deciduous forests, tropical dry-deciduous forests and littoral and tidal swamp forests, as well as mangroves with unique, endemic, rare and endangered floral and faunal species. The climate of Odisha is characterised by tropical monsoon weather as its coast borders the Bay of Bengal. The weather is classified as summer, monsoon and winter. Searing hot summers with considerably high monsoon downpours and cool, pleasant winters mark the Odisha climate. The average rainfall varies from $1200 \mathrm{~mm}$ to $1700 \mathrm{~mm}$ across the state, and is the main source of water. Moreover, the state is vulnerable to multiple disasters such as tropical cyclones, storm surges and tsunamis due to its sub-tropical littoral location (http://nidm.gov.in/default.asp). About 62 ethnic tribal communities have been reported in Odisha, of which 13 are known as "Particularly Vulnerable Tribal Groups" (https://en.wikipedia.org/wiki/List of Scheduled Tri bes in Odisha). Districts such as Kandhamala, Koraput, Malkanigiri, Mayurbhanj, Nabrangpur, Rayagada and Sundargarh have scheduled tribes (officially designated groups of historically disadvantaged people in India) above $50 \%$ of the total population. The social, 
cultural and religious life of aboriginal people is influenced by nature and natural resources available in and around their habitat, which provides their food, medicine, shelter, and various other materials and cultural needs [109,110].

Sasil-Lagoudakis et al. [133] published a review entitled "phylogenies reveal the predictive power of traditional medicine in bioprospecting". Their study, which includes the Asteraceae family, provides unique large-scale evidence that plant bioactivity underlies traditional medicine. According to these authors, "related plants are traditionally used as medicines in different regions, and these plant groups coincide with groups that are used to produce pharmaceutical drugs". The authors conclude that "phylogenetic cross-cultural comparisons can focus screening efforts on a subset of traditionally used plants that are richer in bioactive compounds, and could revitalise the use of traditional knowledge in bioprospecting".

Gertrude et al. [36] studied the anthelmintic activity of Bidens pilosa leaf against Haemonchus contortus eggs and larvae and concluded that ethanolic extracts have the potential to inhibit the growth of Haemonchus contortus. However, further study on the isolation of the active compounds as well as in vivo studies are needed. Similarly, antileishmanial activity of Bidens pilosa leaf was reported by several researchers $[31,85]$, but no compound responsible for this activity has been identified so far. The anthelmintic and wormicidal properties of Blumea lacera leaf were evaluated against Ascaris lumbricoides and Pheretima posthuma [119], but no bioactive compounds have been acknowledged so far. Calendula officinalis has been used traditionally by the tribes of Odisha for worm infections. Nikmehr et al. [95] found that crude methanolic extracts have antileishmanial activity, but no bioactive molecules have been isolated so far. Caesulia axillaris, a wetland plant, is used very frequently for the treatment of malaria by the coastal peoples of Odisha. However, despite its long traditional use, its scientific validation as an antiparasitic agent has not been established so far. Also, the phytochemistry of this plant is not well known, except for a few studies on its essential oils. Similarly, plants such as Centipeda minima, Sphaeranthus indicus and Tagetes erecta are used as anthelmintic plants by the tribes of Odisha for the treatment of worm infections. Yu et al. [164] found antiparasitic activity of crude extracts of Centipeda minima and its fractions against Giardia intestinalis, Entamoeba histolytica and Plasmodium falciparum. Crude extracts of Sphaeranthus indicus also showed antiparasitic effects on Ascaridia galli, Entamoeba histolytica and Setaria digitate $[96,134]$. Organic and aqueous extracts of Tagetes erecta show antiparasitic [41], and anthelmintic properties [106]. However, notwithstanding phytochemical studies, no anti-parasitic compounds have been identified, nor have any in vivo studies been conducted so far on these plants. The plant Elephantopus scaber showed anthelmintic activity against Pheretima posthuma in crude extract. However, further study is required to find out the active anthelmintic compounds. Both in vitro and in vivo studies were carried out and proved the anthelmintic properties of Vernonia anthelmintica against Haemonchus contortus [103,106,140]. Further study is needed to determine the active anthelmintic compounds. The tribes of Odisha frequently use two other species of Vernonia: V. albicans and V. cinerea. These plants are also interesting for future study to discover active molecules with antiparasitic properties. The antitrypanosomal activity of a crude $50 \%$ ethanol extract of Xanthium strumarium leaves was studied in vitro and in vivo. The extract exhibited trypanocidal activity against Trypanosoma evansi-infected mice [147]. The authors hypothesised that the presence of xanthinin may be responsible for its trypanocidal activity, but further study is needed to definitively identify the antitrypanosomal compound or compounds.

\section{Conclusion}

A search for new antiparasitic drugs has been under way over the past several decades. However, despite the abundant literature, more work is needed to yield potent, commercially available drugs based on natural products. Fortunately, academic drug discovery for neglected diseases has intensified (e.g. the Drugs for Neglected Disease Initiative http://www.dndi.org/), and this includes efforts to use natural products (e.g. Research Network Natural Products against Neglected Diseases https://www.facebook.com/ResNetNPND/ app/435433039823956). Although many Asteraceae species were already studied for different antiparasitic activities, some of the species important in traditional medicines have still hardly been studied for their bioactivity. Therefore, the present review aims to encourage further exploration of their potential bioactivity and particularly their antiparasitic properties, guided by the knowledge on the use of Asteraceae plants by the tribes of Odisha and corresponding traditional uses elsewhere in the world. The work reported here highlights the traditional uses of Asteraceae plants of Odisha for the treatment of parasites. Plants such as Bidens pilosa, Blumea lacera, Caesulia axillaris, Centipeda minima and Sphaeranthus indicus deserve to be studied further, especially concerning their most relevant bioactive properties and significant bioactive compounds that could be purified with stateof-the-art methods.

Acknowledgment. The authors are thankful to KU Leuven for providing the necessary facilities during preparation of this review article. This project received funding from the European Union's Horizon 2020 research and innovation programme under Grant agreement No 633589. This publication reflects only the authors' views and the Commission is not responsible for any use that may be made of the information it contains.

\section{Conflict of interest}

The authors declare that they have no conflict of interest. 


\section{References}

1. Aguiar GP, Melo NI, Wakabayashi KAL, Lopes MHS, Mantovani ALL, Dias HJ, Fukui MJ, Keles LC, Rodrigues V, Groppo M, Silva-Filho AA, Cunha WR, Magalhães LG, Crotti AEM. 2013. Chemical composition and in vitro schistosomicidal activity of the essential oil from the flowers of Bidens sulphurea (Asteraceae). Natural Product Research, 27, 920-924.

2. Akendengué B. 1992. Medicinal plants used by the Fang traditional healers in Equatorial Guinea. Journal of Ethnopharmacology, 37, 165-173.

3. Alvarenga SA, Ferreira MJ, Emerenciano V, Cabrol-Bass D. 2001. Chemosystematic studies of natural compounds isolated from Asteraceae: characterization of tribes by principal component analysis. Chemometrics and Intelligent Laboratory Systems, 56, 27-37.

4. Ashok P, Koti BC, Thippeswamy AHM, Tikare VP, Dabadi P, Viswanathaswamy AHM. 2010. Evaluation of antiinflammatory activity of Centratherum anthelminticum (L) Kuntze Seed. Indian Journal of Pharmaceutical Sciences, 72, 697-703.

5. Bailen M, Julio LF, Diaz CE, Sanz J, Martínez-Díaz RA, Cabrera R, Burillo J, Gonzalez-Coloma A. 2013. Chemical composition and biological effects of essential oils from Artemisia absinthium L. cultivated under different environmental conditions. Industrial Crops and Products, 49, 102-107.

6. Bapna S, Adsule S, Shirshat Mahendra S, Jadhav S, Patil LS, Deshmukh RA. 2007. Anti-malarial activity of Eclipta alba against Plasmodium berghei infection in mice. Journal of Communicable Diseases, 39, 91-104.

7. Bartolome AP, Villaseñor IM, Yang W-C., Yang W-C. 2013. Bidens pilosa L. (Asteraceae): Botanical properties, traditional uses, phytochemistry, and pharmacology. Evidence-based Complementary and Alternative Medicine, 340215 .

8. Becker JV, van der Merwe MM, van Brummelen AC, Pillay P, Crampton BG, Mmutlane EM, Parkinson C, van Heerden FR, Crouch NR, Smith PJ, Mancama DT, Maharaj VJ. 2011. In vitro anti-plasmodial activity of Dicoma anomala subsp. gerrardii (Asteraceae): identification of its main active constituent, structure-activity relationship studies and gene expression profiling. Malaria Journal, 10, 295.

9. Berger I, Passreiter CM, Cáceres A, Kubelka W. 2001. Antiprotozoal activity of Neurolaena lobata. Phytotherapy Research, 15, 327-330.

10. Bero J, Hannaert V, Chataigné G, Hérent MF, QuetinLeclercq J. 2011. In vitro antitrypanosomal and antileishmanial activity of plants used in Benin in traditional medicine and bio-guided fractionation of the most active extract. Journal of Ethnopharmacology, 137, 998-1002.

11. Bhinge SD, Hogade MG, Chavan C, Kumbhar M, Chature V. 2010. In vitro anthelmintic activity of herb extract of Eclipta prostrate L. against Pheretima posthuma. Asian Journal of Pharma Clinical Research, 3, 229-230.

12. Bisht VK, Purohit V. 2010. Medicinal and aromatic plants diversity of Asteraceae in Uttarakhand. Nature and Science, 8, 121-128.

13. Brandão MG, Krettli AU, Soares LS, Nery CG, Marinuzzi HC. 1997. Antimalarial activity of extracts and fractions from Bidens pilosa and other Bidens species (Asteraceae) correlated with the presence of acetylene and flavonoid compounds. Journal of Ethnopharmacology, 57, 131-138.

14. Bringmann G, Saeb W, Assi L, François G, Sankara Narayanan A, Peters K, Peters EM. 1997. Betulinic acid:
Isolation from Triphyophyllum peltatum and Ancistrocladus heyneanus, antimalarial activity, and crystal structure of the benzyl ester. Planta Medica, 63, 255-257.

15. Brun R, Don R, Jacobs RT, Wang MZ, Barrett MP. 2011. Development of novel drugs for human African trypanosomiasis. Future Microbiology, 6, 677-691.

16. Byng JW. The flowering plants handbook: a practical guide to families and genera of the world. Plant Gateway Ltd., Hertford, Plant Gateway Ltd., 2004.

17. Calzada F, Meckes M, Cedillo-Rivera R. 1999. Antiamoebic and antigiardial activity of plant flavonoids. Planta Medica, 65, 78-80.

18. Cox PA. 2000. Will tribal knowledge survive the millennium? Science, 287, 44-45.

19. Das M, Mukherjee A. 2014. Elephantopus scaber L.: An overview. Indian Journal Life Science, 4, 51-54.

20. de Almeida LMS, Carvalho LSA de, Gazolla MC, Silva Pinto PL, Silva MPN da, de Moraes J, Da Silva Filho AA. 2016. Flavonoids and sesquiterpene lactones from Artemisia absinthium and Tanacetum parthenium against Schistosoma mansoni worms. Evidence-Based Complementary and Alternative Medicine, 9521349.

21. Elford BC, Roberts MF, Phillipson JD, Wilson RJM. 1987. Potentiation of the antimalarial activity of qinghaosu by methoxylated flavones. Transactions of the Royal Society of Tropical Medicine and Hygiene, 81, 434436.

22. Enwerem NM, Okogun JI, Wambebe CO, Okorie DA, Akah PA. 2001. Anthelmintic activity of the stem bark extracts of Berlina grandiflora and one of its active principles, Betulinic acid. Phytomedicine, 8, 112-114.

23. Ezenyi IC, Salawu OA, Kulkarni R, Emeje M. 2014. Antiplasmodial activity-aided isolation and identification of quercetin-4'-methyl ether in Chromolaena odorata leaf fraction with high activity against chloroquine-resistant Plasmodium falciparum. Parasitology Research, 113, 4415-4422.

24. Faizi S, Fayyaz S, Bano S, Yawar Iqbal E, Siddiqi H, Naz A, Naz A. 2011. Isolation of nematicidal compounds from Tagetes patula L. yellow flowers: Structure-activity relationship studies against cyst nematode Heterodera zeae infective stage larvae. Journal of Agricultural and Food Chemistry, 59, 9080-9093.

25. Feyera T, Terefe G, Shibeshi W. Evaluation of In vivo antitrypanosomal activity of crude extracts of Artemisia abyssinica against a Trypanosoma congolense isolate. BMC Complementary and Alternative Medicine, 14, 117.

26. Foster JG, Cassida KA, Turner KE. 2011. In vitro analysis of the anthelmintic activity of forage chicory (Cichorium intybus L.) sesquiterpene lactones against a predominantly Haemonchus contortus egg population. Veterinary Parasitology, 180, 298-306.

27. Fournet A, Barrios AA, Muñoz V. 1994. Leishmanicidal and trypanocidal activities of Bolivian medicinal plants. Journal of Ethnopharmacology, 41, 19-37.

28. François G, Passreiter CM, Woerdenbag HJ, Van Looveren M. 1996. Antiplasmodial activities and cytotoxic effects of aqueous extracts and sesquiterpene lactones from Neurolaena lobata. Planta Medica, 62, 126-129.

29. Freiburghaus F, Ogwal EN, Nkunya MH, Kaminsky R, Brun R. 1996. In vitro antitrypanosomal activity of African plants used in traditional medicine in Uganda to treat sleeping sickness. Tropical Medicine and International Health, 1, 765-771.

30. Funk VA, Bayer RJ, Keeley S, Chan R, Watson L, Gemeinholzer B, Schilling E, Panero JL, Baldwin BG, Garcia-Jacas N, Susanna A, Jansen RK. Everywhere but 
Antarctica: Using a supertree to understand the diversity and distribution of the Compositae. Biologiske Skrifter, $55,343-374$.

31. Gachet MS, Lecaro JS, Kaiser M, Brun R, Navarrete H, Muñoz RA, Bauer R, Schuhly W. 2010. Assessment of anti-protozoal activity of plants traditionally used in Ecuador in the treatment of leishmaniasis. Journal of Ethnopharmacology, 128, 184-197.

32. Gallwitz H, Bonse S, Martinez-Cruz A, Schlichting I, Schumacher K, Krauth-Siegel RL. 1999. Ajoene is an inhibitor and subversive substrate of human glutathione reductase and Trypanosoma cruzi trypanothione reductase: Crystallographic, kinetic, and spectroscopic studies. Journal of Medicinal Chemistry, 42, 364-372.

33. Gamboa-Leon R, Vera-Ku M, Peraza-Sanchez SR, KuChulim C, Horta-Baas A, Rosado-Vallado M. 2014. Antileishmanial activity of a mixture of Tridax procumbens and Allium sativum in mice. Parasite, 21, 15.

34. Ganfon H, Bero J, Tchinda AT, Gbaguidi F, Gbenou J, Moudachirou M, Frédérich M, Quetin-Leclercq J. 2012. Antiparasitic activities of two sesquiterpenic lactones isolated from Acanthospermum hispidum D.C. Journal of Ethnopharmacology, 141, 411-417.

35. García M, Monzote L, Scull R, Herrera P. 2012. Activity of Cuban plants extracts against Leishmania amazonensis. ISRN Pharmacology, 104540.

36. Gertrude Mbogning Tayo, Payne VK, Poné JW, Claire KM, Jeannette Y, Alidou MN, Mbida M, Bilong CFB. 2012. In vitro ovicidal and larvicidal activities of aqueous and ethanolic extracts of the leaves of Bidens pilosa (Asteraceae) on Heligmosomoides bakeri (Nematoda: Heligmosomatidae). International Journal of Phytomedicines and Related Industries, 4, 121-125.

37. Girach RD, Brahmam M, Misra MK, Ahmed M. 1998. Indigenous phytotherapy for filariasis from Orissa. Ancient Science of Life, 17, 224-227.

38. Goffin E, da Cunha AP, Ziemons E, Tits M, Angenot L, Frederich M. 2003. Quantification of tagitinin C in Tithonia diversifolia by reversed-phase high-performance liquid chromatography. Phytochemical Analysis, 14, 378380 .

39. Gogate G, Ananthasubramanian L, Nargund KS, Bhattacharya SC. 1986. Some interesting sesqueterpenoids from Sphaeranthus indicus Linn. Indian Journal of Chemistry, 25, 233-238.

40. Grecco SS, Reimão JQ, Tempone AG, Sartorelli P, Cunha RLOR, Romoff P, Ferreira MJP, Fávero OA, Lago JHG. 2012. In vitro antileishmanial and antitrypanosomal activities of flavanones from Baccharis retusa DC. (Asteraceae). Experimental Parasitology, 130, 141-145.

41. Gupta P, Vasudeva N. 2010. In vitro antiplasmodial and antimicrobial potential of Tagetes erecta roots. Pharmaceutical Biology, 48, 1218-1223.

42. Gurib-Fakim A. 2006. Medicinal plants: Traditions of yesterday and drugs of tomorrow. Molecular Aspects of Medicine 27, 1-93.

43. Hatimi S, Boudouma M, Bichichi M, Chaib N, Idrissi NG. 2001. In vitro evaluation of antileishmania activity of Artemisia herba alba Asso. Bulletin de la Société de Pathologie Exotique, 94, 29-31.

44. Hördegen P, Cabaret J, Hertzberg H, Langhans W, Maurer V. 2006. In vitro screening of six anthelmintic plant products against larval Haemonchus contortus with a modified methyl-thiazolyl-tetrazolium reduction assay. Journal of Ethnopharmacology, 108, 85-89.

45. Iqbal Z, Lateef M, Jabbar A, Akhtar MS, Khan MN. 2006. Anthelmintic activity of Vernonia anthelmintica seeds against Trichostrongylid nematodes of sheep. Pharmaceutical Biology, 44, 563-567.

46. Islam MT, Das PR, Kabir MH, Akter S, Khatun Z, Haque MM, Roney MSI, Jahan R, Rahmatullah M. 2012. Acanthaceae and Asteraceae family plants used by folk medicinal practitioners for treatment of malaria in Chittagong and Sylhet divisions of Bangladesh. AmericanEurasian Journal of Sustainable Agriculture, 6, 146-152.

47. Jahan R, Al-Nahain A, Majumder S, Rahmatullah M. 2014. Ethnopharmacological Significance of Eclipta alba (L.) Hassk. (Asteraceae). International Scholarly Research Notices, 385969.

48. Jansen O, Tits M, Angenot L, Nicolas JP, Patrick De Mol JBN, Frédérich M. 2012. Anti-plasmodial activity of Dicoma tomentosa (Asteraceae) and identification of urospermal A-15- $\mathrm{O}$-acetate as the main active compound. Malaria Journal, 11, 289.

49. Jesus JA, Fragoso TN, Yamamoto ES, Laurenti MD, Silva MS, Ferreira AF, Lago JHG, Gomes GS, Passero LFD. 2017. Therapeutic effect of ursolic acid in experimental visceral leishmaniasis. International Journal for Parasitology: Drugs and Drug Resistance, 7, 1-11.

50. Kamaraj C, Rahuman AA. 2011. Efficacy of anthelmintic properties of medicinal plant extracts against Haemonchus contortus. Research in Veterinary Science, 91, 400-404.

51. Kamboj A, Saluja AK. 2008. Ageratum conyzoides L.: A review on its phytochemical and pharmacological profile. International Journal of Green Pharmacy, 2, 59-68.

52. Kamboj A, Saluja AK. 2010. Phytopharmacological review of Xanthium strumarium L. (Cocklebur). International Journal of Green Pharmacy, 4, 129-139.

53. Kantamreddi VSS, Parida S, Kommula SM, Wright CW. 2009. Phytotherapy used in Orissa state, India for treating malaria. Phytotherapy Research, 23, 1638-1641.

54. Kayser O, Kiderlen AF, Folkens U, Kolodziej H. 1999. In vitro leishmanicidal activity of Aurones. Planta Medica, 65, 316-319.

55. Kimura Y, Hiraoka K, Kawano T, Fujioka S, Shimada A. 2017. Nematicidal activities of acetylene compounds from Coreopsis lanceolata L. Zeitschrift fur Naturforschung $\mathrm{C}, 63,843-847$.

56. Khanna VG, Kannabiran K, Getti G. 2009. Leishmanicidal activity of saponins isolated from the leaves of Eclipta prostrata and Gymnema sylvestre. Indian Journal of Pharmacology, 41, 32-35.

57. Klongsiriwet C, Quijada J, Williams AR, Mueller-Harvey I, Williamson EM, Hoste H. 2015. Synergistic inhibition of Haemonchus contortus exsheathment by flavonoid monomers and condensed tannins. International Journal for Parasitology: Drugs and Drug Resistance, 5, 127-134.

58. Koide T, Nose M, Inoue M, Ogihara Y, Yabu Y, Ohta N. 1998. Trypanocidal effects of gallic acid and related compounds. Planta Medica, 64, 27-30.

59. Komlaga G, Agyare C, Dickson RA, Mensah MLK, Annan K, Loiseau PM, et al. 2015. Medicinal plants and finished marketed herbal products used in the treatment of malaria in the Ashanti region, Ghana. Journal of Ethnopharmacology, 172, 333-436.

60. Koshimizu K, Ohigashi H, Huffman MA. Use of Vernonia amygdalina by wild chimpanzee: possible roles of its bitter and related constituents. Physiology and Behavior, 56, $1209-1216$.

61. Kovendan K, Arivoli S, Maheshwaran R, Baskar K, Vincent S. 2012. Larvicidal efficacy of Sphaeranthus indicus, Cleistanthus collinus and Murraya koenigii leaf extracts against filarial vector, Culex quinquefasciatus Say (Diptera: Culicidae). Parasitology Research, 111, 1025-1035. 
62. Kuhn T, Wang Y. 2008. Artemisinin-an innovative cornerstone for anti-malaria therapy. Progress in Drug Research, 66, 385-422.

63. Kumari P, Misra K, Sisodia B, Faridi U, Srivastava S, Luqman S, Darokar M, Negi A, Gupta M, Singh S, Kumar J. 2009. A promising anticancer and antimalarial component from the leaves of Bidens pilosa. Planta Medica, 75, $59-61$.

64. Lans C, Turner N, Khan T, Brauer G. 2007. Ethnoveterinary medicines used to treat endoparasites and stomach problems in pigs and pets in British Columbia, Canada. Veterinary Parasitology, 148, 325-340.

65. Lavault M, Landreau A, Larcher G, Bouchara J-P, Pagniez F, Le Pape P, Richomme P. 2005. Antileishmanial and antifungal activities of xanthanolides isolated from Xanthium macrocarpum. Fitoterapia, 76, 363-366.

66. Lehane AM, Saliba KJ. 2008. Common dietary flavonoids inhibit the growth of the intraerythrocytic malaria parasite. BMC Research Notes, 1, 26.

67. Lima Silva F, Fischer DCH, Fechine-Tavares J, SobralSilva M, Filgueiras de Athayde-Filho P, Barbosa-Filho JM. 2011. Compilation of secondary metabolites from Bidens pilosa L. Molecules 16, 1070-1102.

68. Lin YL, Chang CC, Lee IJ. 2008. Review on phytochemical study of Asteraceae in Taiwan (1996-2005). Journal of Chinese Medicine, 1934, 135-149.

69. Lockman Y, Vardy D, Ohayon D, el-On J. 1991. The failure of traditionally used desert plants to act against cutaneous leishmaniasis in experimental animals. Annals of Tropical Medicine and Parasitology, 85, 499-501.

70. Lozano R, Naghavi M, Foreman K, Lim S, Shibuya K, Aboyans V, Abraham J, Adair T, Aggarwal R, Ahn SY, AlMazroa MA, Alvarado M, Anderson HR, Anderson LM, Andrews KG, Atkinson C, Baddour LM, Barker-Collo S, Bartels DH, Bell ML, Benjamin EJ, Bennett D, Bhalla K, Bikbov B, Abdulhak A Bin, Birbeck G, Blyth F, Bolliger I, Boufous S, Bucello C, Burch M, Burney P, Carapetis J, Chen H, Chou D, Chugh SS, Coffeng LE, Colan SD, Colquhoun S, Colson KE, Condon J, Connor MD, Cooper LT, Corriere M, Cortinovis M, de Vaccaro KC, Couser W, Cowie BC, Criqui MH, Cross M, Dabhadkar KC, Dahodwala N, De Leo D, Degenhardt L, Delossantos A, Denenberg J, Des Jarlais DC, Dharmaratne SD, Dorsey ER, Driscoll T, Duber H, Ebel B, Erwin PJ, Espindola P, Ezzati M, Feigin V, Flaxman AD, Forouzanfar MH, Fowkes FGR, Franklin R, Fransen M, Freeman MK, Gabriel SE, Gakidou E, Gaspari F, Gillum RF, GonzalezMedina D, Halasa YA, Haring D, Harrison JE, Havmoeller R, Hay RJ, Hoen B, Hotez PJ, Hoy D, Jacobsen KH, James SL, Jasrasaria R, Jayaraman S, Johns N, Karthikeyan G, Kassebaum N, Keren A, Khoo J-P, Knowlton LM, Kobusingye O, Koranteng A, Krishnamurthi R, Lipnick M, Lipshultz SE, Ohno SL, Mabweijano J, MacIntyre MF, Mallinger L, March L, Marks GB, Marks R, Matsumori A, Matzopoulos R, Mayosi BM, McAnulty JH, McDermott MM, McGrath J, Memish ZA, Mensah GA, Merriman TR, Michaud C, Miller M, Miller TR, Mock C, Mocumbi AO, Mokdad AA, Moran A, Mulholland K, Nair MN, Naldi L, Narayan KMV, Nasseri K, Norman P, O'Donnell M, Omer SB, Ortblad K, Osborne R, Ozgediz D, Pahari B, Pandian JD, Rivero AP, Padilla RP, Perez-Ruiz F, Perico N, Phillips D, Pierce K, Pope CA, Porrini E, Pourmalek F, Raju M, Ranganathan D, Rehm JT, Rein DB, Remuzzi G, Rivara FP, Roberts T, De León FR, Rosenfeld LC, Rushton L, Sacco RL, Salomon JA, Sampson U, Sanman E, Schwebel DC, Segui-Gomez M, Shepard DS, Singh D, Singleton J, Sliwa K, Smith E, Steer A, Taylor JA, Thomas
B, Tleyjeh IM, Towbin JA, Truelsen T, Undurraga EA, Venketasubramanian N, Vijayakumar L, Vos T, Wagner GR, Wang M, Wang W, Watt K, Weinstock MA, Weintraub R, Wilkinson JD, Woolf AD, Wulf S, Yeh PH, Yip P, Zabetian A, Zheng Z-J, Lopez AD, Murray CJ. 2012. Global and regional mortality from 235 causes of death for 20 age groups in 1990 and 2010: a systematic analysis for the global burden of disease study 2010. Lancet, 380, 2095-2128.

71. Mahajan NG, Chopda MZ, Mahajan RT. 2015. A review on Sphaeranthus indicus Linn: Multipotential medicinal plant. International Journal of Pharmaceutical Research and Allied Sciences, 4, 48-74.

72. Maldonado EM, Salamanca E, Giménez A, Saavedra G, Sterner O. 2014. Antileishmanial metabolites from Trixis antimenorrhoea. Phytochemistry Letters, 10, 281-286.

73. Martín T, Villaescusa L, Gasquet M, Delmas F, Bartolomé C, Díaz-Lanza AM, Ollivier E, Balansard G. 1998. Screening for protozoocidal activity of Spanish plants. Pharmaceutical Biology, 36, 56-62.

74. Martínez-Díaz RA, Ibáñez-Escribano A, Burillo J, De Las Heras L, Del Prado G, Agulló-Ortuño MT, Julio LF, González-Coloma A. 2015. Trypanocidal, trichomonacidal and cytotoxic components of cultivated Artemisia absinthium Linnaeus (Asteraceae) essential oil. Memorias do Instituto Oswaldo Cruz, 110, 693-699.

75. Martín-Quintal Z, Moo-Puc R, González-Salazar F, Chan-Bacab MJ, Torres-Tapia LW, Peraza-Sánchez SR. 2009. In vitro activity of Tridax procumbens against promastigotes of Leishmania mexicana. Journal of Ethnopharmacology, 122, 463-467.

76. Martio-Quintal Z, del Rosario Garcia-Miss M, MutMartin M, Matus-Moo A, Torres-Tapia LW, PerazaSanchez SR. 2009. The leishmanicidal effect of (3 S)-16, 17-didehydrofalcarinol, an oxylipin isolated from Tridax procumbens, is independent of NO production. Phytotherapy Research, 24, 1004-1008.

77. McChesney JD, Venkataraman SK, Henri JT. 2007. Plant natural products: Back to the future or into extinction? Phytochemistry, 68, 2015-2022.

78. Mead JR, McNair N. 2006. Antiparasitic activity of flavonoids and isoflavones against Cryptosporidium parvum and Encephalitozoon intestinalis. FEMS Microbiology Letters, 259, 153-157.

79. Meckes M, Calzada F, Tapia-Contreras A, Cedillo-Rivera R. 1999. Antiprotozoal properties of Helianthemum glomeratum. Phytotherapy Research, 13, 102-105.

80. Mishra P, Kumar Mishra S, lacera Pratibha Mishra BD, Irchhiaya R. 2015. Phytochemical investigation and spectral studies of isolated flavonoid from ethanolic extract of whole plant. Journal of Pharmacognosy and Phytochemistry, 4, 1-4.

81. Mohanty N, Panda T, Sahoo S, Rath SP. 2015. Herbal folk remedies of Dhenkanal district, Odisha, India. International Journal of Herbal Medicine, 3, 24-33.

82. Mokoka T, Zimmermann S, Julianti T, Hata Y, Moodley N, Cal M, Adams M, Kaiser M, Brun R, Koorbanally N, Hamburger M. 2011. In vitro screening of traditional South African malaria remedies against Trypanosoma brucei rhodesiense, Trypanosoma cruzi, Leishmania donovani and Plasmodium falciparum. Planta Medica, 77, 1663-1667.

83. Morais TR, Romoff P, Fávero OA, Reimão JQ, Lourenço WC, Tempone AG, Hristov AD, Di Santi SM, Lago JHG, Sartorelli P, Ferreira MJP. 2012. Anti-malarial, antitrypanosomal, and anti-leishmanial activities of jacaranone isolated from Pentacalia desiderabilis (Vell.) Cuatrec. (Asteraceae). Parasitology Research, 110, 95-101. 
84. Moshi MJ, Otieno DF, Mbabazi PK, Weisheit A. 2010. Ethnomedicine of the Kagera Region, North Western Tanzania. Part 2: The medicinal plants used in Katoro Ward, Bukoba District. Journal of Ethnobiology and Ethnomedicine, 6, 19

85. Moyo P, Botha ME, Nondaba S, Niemand J, Maharaj VJ, Eloff JN, Louw AI, Birkholtz L. 2016. In vitro inhibition of Plasmodium falciparum early and late stage gametocyte viability by extracts from eight traditionally used South African plant species. Journal of Ethnopharmacology, 185, 235-242.

86. Mujtaba Shah G, Abbasi AM, Khan N, Guo X, Ajab Khan M, Hussain M, Bibi S, Nazir A, Ahmad Tahir A. 2014 Traditional uses of medicinal plants against malarial disease by the tribal communities of Lesser HimalayasPakistan. Journal of Ethnopharmacology, 155, 450-462.

87. Muley B, Khadabadi S, Banarase N. 2009. Phytochemical constituents and pharmacological activities of Calendula officinalis Linn (Asteraceae): A review. Tropical Journal of Pharmaceutical Research, 8, 455-465.

88. Murray CJL, Rosenfeld LC, Lim SS, Andrews KG, Foreman KJ, Haring D, Fullman N, Naghavi M, Lozano R, Lopez AD. 2012. Global malaria mortality between 1980 and 2010: a systematic analysis. Lancet, 379, 413-431.

89. Musayeib N, Mothana R, Gamal A, Al-Massarani S, Maes L. 2013. In vitro antiprotozoal activity of triterpenoid constituents of Kleinia Odora growing in Saudi Arabia. Molecules, 18, 9207-9218.

90. Muthaura CN, Keriko JM, Mutai C, Yenesew A, Gathirwa JW, Irungu BN, Nyangacha R, Mungai GM, Derese S. 2015. Antiplasmodial potential of traditional antimalarial phytotherapy remedies used by the Kwale community of the Kenyan Coast. Journal of Ethnopharmacology, 170, 148-157.

91. Ndjakou Lenta B, Devkota KP, Ngouela S, Fekam Boyom F, Naz Q, Choudhary MI, Tsamo E, Rosenthal PJ, Sewald N. 2008. Anti-plasmodial and cholinesterase inhibiting activities of some constituents of Psorospermum glaberrimum. Chemical and Pharmaceutical Bulletin, 56, 222-226.

92. Newman DJ, Cragg GM. 2016. Natural products as sources of new drugs from 1981 to 2014. Journal of Natural Products, 79, 629-661.

93. Niaz S, Akhtar T, Shams S, AbdEl-Salam NM, Ayaz S, Ullah R, Bibi S, Hussain I, Ahmad S. 2015. Treatment of bovine schistosomiasis with medicinal plant, Veronia anthelmintica (Kaliziri), an alternative approach. African Journal of Traditional, Complementary and Alternative Medicines, 12S, 78-83.

94. Nibret E, Wink M. 2010. Volatile components of four Ethiopian Artemisia species extracts and their In vitro antitrypanosomal and cytotoxic activities. Phytomedicine, 17, 369-374.

95. Nikmehr B, Ghaznavi H, Rahbar A, Sadr S, Mehrzadi S. 2014. In vitro anti-leishmanial activity of methanolic extracts of Calendula officinalis flowers, Datura stramonium seeds, and Salvia officinalis leaves. Chinese Journal of Natural Medicines, 12, 423-427.

96. Nisha M, Kalyanasundaram M, Paily KP, Abidha, Vanamail P, Balaraman K. 2006. In vitro screening of medicinal plant extracts for macrofilaricidal activity. Parasitology Research, 100, 575-579.

97. Nour A, Khalid S, Abdallah W, Kaiser M, Brun R, Schmidt T. 2006. Trypanocidal flavonoids from Ageratum conyzoides. Planta Medica, $72, \mathrm{P} \quad 073$

98. Nour AMM, Khalid SA, Kaiser M, Brun R, Abdalla WE, Schmidt TJ. 2010. The antiprotozoal activity of methylated flavonoids from Ageratum conyzoides L. Journal of Ethnopharmacology, 129, 127-130.
99. Nweze N, Anene B, Asuzu I. 2011. African Journal of Biotechnology, 10, 15626-15632.

100. Ohigashi H, Huffman MA, Izutsu D, Koshimizu K, Kawanaka M, Sugiyama H, Kirby GC, Warhurst DC, Allen D, Wright CW, David Phillipson J, Timon-David P, Delmas F, Elias R, Balansard G. 1994. Toward the chemical ecology of medicinal plant use in chimpanzees: The case of Vernonia amygdalina, a plant used by wild chimpanzees possibly for parasite-related diseases. Journal of Chemical Ecology, 20, 541-553.

101. Oketch-Rabah HA, Lemmich E, Dossaji SF, Theander TG, Olsen CE, Cornett IC, Arsalan KV, Christensen SV. 1997. Two new antiprotozoal 5-Methylcoumarins from Vernonia brachycalyx. Journal of Natural Products, 60, 458-461.

102. Oliveira F, Andrade-Neto V, Krettli A, Brandão MG. 2004. New evidences of antimalarial activity of Bidens pilosa roots extract correlated with polyacetylene and flavonoids. Journal of Ethnopharmacology, 93, 39-42.

103. Olukunle JO, Jacobs EB, Oyewusi JA, Durotoye LA. 2015. Effects of aqueous leaf extract of Tithonia diversifolia (Mexican Sunflower) on semen characteristic and morphology in male Wistar albino rats. Nigerian Journal of Animal Production, 42, 263-270.

104. Oryan A, Akbari M. 2016. Worldwide risk factors in leishmaniasis. Asian Pacific Journal of Tropical Medicine, 9, 925-932.

105. Oudhia P, Tripathi RS. 1974. A useful weed Blumea lacera L. - A review. Indian Journal of Weed Science, 31, 108-109.

106. Palacios Landín J, Mendoza de Gives P, Salinas Sánchez DO, López Arellano ME, Liébano Hernández E, Hernández Velázquez VM, Valladares Cisneros MG. 2016. In vitro and in vivo nematocidal activity of Allium sativum and Tagetes erecta extracts against Haemonchus contortus. Turkish Journal of Parasitology, 39, 260-264.

107. Panda A, Misra MK. 2011. Ethnomedicinal survey of some wetland plants of South Orissa and their conservation. Indian Journal of Traditional Knowledge, 10, 296-303.

108. Panda SK, Das D, Tripthathy NK. 2015. Phytochemical investigation and anthelmintic activity of various root extracts of Gmelina arborea. Asian Journal of Plant Science and Research, 5, 54-58.

109. Panda SK, Mohanta YK, Padhi L, Park Y-H., Mohanta TK, Bae H. 2016. Large scale screening of ethnomedicinal plants for identification of potential antibacterial compounds. Molecules, 21, 293.

110. Panda SK, Padhi L, Leyssen P, Liu M, Neyts J, Luyten W. 2017. Antimicrobial, anthelmintic and antiviral activity of plants traditionally used for treating infectious disease in the Similipal Biosphere Reserve, Odisha, India. Frontiers in Pharmacology, 8, 658 .

111. Panda SK, Rout SD, Mishra N, Panda T. 2011. Phytotherapy and traditional knowledge of tribal communities of Mayurbhanj district, Orissa, India. Journal of Pharmacognosy and Phytotherapy, 3, 101-113.

112. Panda SK. 2014. Ethno-medicinal uses and screening of plants for antibacterial activity from Similipal Biosphere Reserve, Odisha, India. Journal of Ethnopharmacology, 151, 158-175

113. Pani M, Nahak G, Sahu RK. 2014. Review on ethnomedicinal plants of Odisha for the treatment of malaria. International Journal of Pharmacognosy and Phytochemical Research, 7, 156-165.

114. Parnham MJ. 1996. Benefit-risk assessment of the squeezed sap of the purple coneflower (Echinacea purpurea) for long-term oral immunostimulation. Phytomedicine, 3, 95-102 
115. Parveen S, Godara R, Katoch R, Yadav A, Verma PK, Katoch M, Singh NK. 2014. In vitro evaluation of ethanolic extracts of Ageratum conyzoides and Artemisia absinthium against cattle tick Rhipicephalus microplus. Scientific World Journal, 858973.

116. Passero LFD, Bonfim-Melo A, Corbett CEP, Laurenti MD, Toyama MH, de Toyama DO, Romoff P, Fávero OA, dos Grecco SS, Zalewsky CA, Lago JHG. 2011. Antileishmanial effects of purified compounds from aerial parts of Baccharis uncinella C. DC. (Asteraceae). Parasitology Research, 108, 529-536.

117. Pattanaik C, Reddy C, Dhal N. 2008. Phytomedicinal study of coastal sand dune species of Orissa. Indian Journal of Traditional Knowledge, 7, 263-268.

118. Pattanayak B, Dhal NK, Parida S. 2016. Ethnobotanical survey of antimalarial plants of Odisha, India. Asian Journal of Science and Technology, 7, 2529-2536.

119. Pattewar AM, Dawalbaje AB, Gundale DM, Pawar PB, Kavtikwar PG, Yerawar PP, Pandharkar TM, Patawar VA. 2012. Phytochemistry and anthelmintic studies on Blumea lacera. Indo Global Journal of Pharmaceutical Sciences, 2, 390-396.

120. Pattnaik A, Sarkar R, Sharma A, Yadav KK, Kumar A, Roy P, Mazumder A, Karmakar S, Sen T. 2013. Pharmacological studies on Buchanania lanzan Spreng. A focus on wound healing with particular reference to antibiofilm properties. Asian Pacific Journal of Tropical Biomedicine, 3, 967-974.

121. Pedersen MM, Chukwujekwu JC, Lategan CA, Staden J van, Smith PJ, Staerk D. 2009. Antimalarial sesquiterpene lactones from Distephanus angulifolius. Phytochemistry, 70, 601-607.

122. Pérez-Victoria JM, Chiquero MJ, Conseil G, Dayan G, Di Pietro A, Barron D, Castanys S, Gamarro F. 1999. Correlation between the affinity of flavonoids binding to the cytosolic site of Leishmania tropica multidrug transporter and their efficiency to revert parasite resistance to Daunomycin. Biochemistry, 38, 1736-1743.

123. Picman AK, Rodriguez E, Towers GH. 1979. Formation of adducts of parthenin and related sesquiterpene lactones with cysteine and glutathione. Chemico-biological Interactions, 28, 83-89.

124. Pink R, Hudson A, Mouriès MA, Bendig M. 2005. Opportunities and challenges in antiparasitic drug discovery. Nature Reviews Drug Discovery, 4, 727-740.

125. Ramachandran S. 2013. Review on Sphaeranthus indicus Linn. (Koṭtaikkarantai). Pharmacognosy Reviews, 7, 157-169.

126. Ramawat KG, Goyal S. 2008. The Indian herbal drugs scenario in global perspectives, in Bioactive molecules and medicinal plants, Ramawat K, Merillon J, Editors. Springer, Berlin, Heidelberg, p. 325-347.

127. Reale S, Pace L, Monti P, Angelis F De Marcozzi G. 2008. A rapid method for the quantification of artemisinin in Artemisia annua L. plants cultivated for the first time in Burundi. Natural Product Research, 22, 360-364.

128. Rodriguez E, Aregullin M, Nishida T, Uehara S, Wrangham R, Abramowski Z, Finlayson A, Towers GH. 1985. Thiarubrine A, a bioactive constituent of Aspilia (Asteraceae) consumed by wild chimpanzees. Experientia, 41, 419-420.

129. Rodríguez-Chávez JL, Rufino-González Y, Ponce-Macotela M, Delgado G. 2015. In vitro activity of "Mexican Arnica" Heterotheca inuloides Cass natural products and some derivatives against Giardia intestinalis. Parasitology, $142,576-584$.
130. Rout SD, Panda SK. 2010. Ethnomedicinal plant resources of Mayurbhanj district, Orissa. Indian Journal of Traditional Knowledge, 9, 68-72.

131. Saini A, Kumar Soni H, Gupta P. 2016. A Review on Tridax procumbens. Imperial Journal of Interdisciplinary Research, 2, 308-319.

132. Sangsopha W, Lekphrom R, Kanokmedhakul S, Kanokmedhakul K. 2016. Cytotoxic and antimalarial constituents from aerial parts of Sphaeranthus indicus. Phytochemistry Letters, 17, 278-281.

133. Saslis-Lagoudakis CH, Savolainen V, Williamson EM, Forest F, Wagstaff SJ, Baral SR, Watson MF, Pendry CA, Hawkins JA. 2012. Phylogenies reveal predictive power of traditional medicine in bioprospecting. Proceedings of the National Academy of Sciences, 109, 15835-15840.

134. Sharma S, Jalalpure SS, Bhupesh S, Shruti T, Agarwal N. 2011. Anthelminitic activity of the whole plant of Sphaeranthus indius. International Journal of Ayurvedic and Herbal Medicine, 1, 14-21.

135. Shetty LJ, Sakr FM, Al-Obaidy K, Patel MJ, Shareef H. 2015. A brief review on medicinal plant Tagetes erecta Linn A. Journal of Applied Pharmaceutical Science, 5, 9195.

136. Shukla AN, Rawat AKS. 2010. An ethnobotanical study of medicinal plants of Rewa district, Madhya Pradesh. Indian Journal of Traditional Knowledge, 9, 191-202.

137. Silveira N, Saar J, Santos A, Barison A, Sandjo L, Kaiser M, Schmidt T, Biavatti M. 2016. A new alkamide with an endoperoxide structure from Acmella ciliata (Asteraceae) and its In vitro antiplasmodial activity. Molecules, 21, 765.

138. Singh SK, Bimal S, Narayan S, Jee C, Bimal D, Das P, Bimal R. 2011. Leishmania donovani: Assessment of leishmanicidal effects of herbal extracts obtained from plants in the visceral leishmaniasis endemic area of Bihar, India. Experimental Parasitology, 127, 552-558.

139. Sirama V, Kokwaro JOwuar B, Yusuf A. 2014. In vitro anthelmintic bioactivity study of Eclipta prostrata L. (whole plant) using adult Haemonchus contortus worms: A case study of Migori County, Kenya. IOSR Journal of Pharmacy and Biological Sciences Ver, 9, 45-53.

140. Soejarto DD, Fong HHS, Tan GT, Zhang HJ, Ma CY, Franzblau SG, Gyllenhaal C, Riley MC, Kadushin MR, Pezzuto JM, Xuan LT, Hiep NT, Hung NV, Vu BM, Loc PK, Dac LX, Binh LT, Chien NQ, Hai NV, Bich TQ, Cuong NM, Southavong B, Sydara K, Bouamanivong S, Ly HM, Thuy TV, Rose WC, Dietzman GR. 2005. Ethnobotany/ethnopharmacology and mass bioprospecting: Issues on intellectual property and benefit-sharing. Journal of Ethnopharmacology, 100, 15-22.

141. Steele JCP, Warhurst DC, Kirby GC, Simmonds MSJ. 1999. In vitro and In vivo evaluation of betulinic acid as an antimalarial. Phytotherapy Research, 13, 115-119.

142. Subhuti D. 2000. Bidens: A popular remedy escapes notice of Western Practitioners. ITM, India.

143. Sülsen VP, Puente V, Papademetrio D, Batlle A, Martino VS, Frank FM, Lombardo ME. 2016. Mode of action of the sesquiterpene lactones psilostachyin and psilostachyin $\mathrm{C}$ on Trypanosoma cruzi. Plos One, 11, e0150526.

144. Sutherland CJ, Tanomsing N, Nolder D, Oguike M, Jennison C, Pukrittayakamee S, Dolecek C, Hien TT, do Rosário VE, Arez AP, Pinto J, Michon P, Escalante AA, Nosten F, Burke M, Lee R, Blaze M, Otto TD, Barnwell JW, Pain A, Williams J, White NJ, Day NPJ, Snounou G, Lockhart PJ, Chiodini PL, Imwong M, Polley SD. 2010. 
Two nonrecombining sympatric forms of the human malaria parasite Plasmodium ovale occur globally. Journal of Infectious Diseases, 201, 1544-1550.

145. Szakiel A, Ruszkowski D, Grudniak A, Kurek A, Wolska K, Doligalska M, Janiszowska W. 2008. Antibacterial and antiparasitic activity of oleanolic acid and its glycosides isolated from marigold (Calendula officinalis). Planta Medica, 74, 1709-1715.

146. Takahashi HT, Novello CR, Ueda-Nakamura T, Filho BPD, Palazzo de Mello JC, Nakamura CV. 2011. Thiophene derivatives with antileishmanial activity isolated from aerial parts of Porophyllum ruderale (Jacq.). Cass. Molecules, 16, 3469-3478.

147. Talakal TS, Dwivedi SK, Sharma SR. 1995. In vitro and In vivo antitrypanosomal activity of Xanthium strumarium leaves. Journal of Ethnopharmacology, 49, 141-145.

148. Tasdemir D, Kaiser M, Brun R, Yardley V, Schmidt TJ, Tosun F, Ruedi P. 2006. Antitrypanosomal and antileishmanial activities of flavonoids and their analogues: In vitro, In vivo, structure-activity relationship, and quantitative structure-activity relationship studies. Antimicrobial Agents and Chemotherapy, 50, 1352-1364.

149. Teixeira TL, Teixeira SC, Silva CV da, Souza MA de. 2014. Potential therapeutic use of herbal extracts in trypanosomiasis. Pathogens and Global Health, 108, 30-36.

150. Tiuman TS, Ueda-Nakamura T, Garcia Cortez DA, Dias Filho BP, Morgado-Díaz JA, de Souza W, Nakamura CV. 2005. Antileishmanial activity of parthenolide, a sesquiterpene lactone isolated from Tanacetum parthenium. Antimicrobial Agents and Chemotherapy, 49, $176-182$.

151. Tobinaga S, Sharma M, Aalbersberg W, Watanabe K, Iguchi K, Narui K, Sasatsu M, Waki S. 2009. Isolation and identification of a potent antimalarial and antibacterial polyacetylene from Bidens pilosa. Planta Medica, 75, 624628.

152. Toyang NJ, Verpoorte R. 2013. A review of the medicinal potentials of plants of the genus Vernonia (Asteraceae). Journal of Ethnopharmacology, 146, 681-723.

153. Tran Q Le Tezuka Y, Ueda J, Nguyen NT, Maruyama Y, Begum K, Kim H-S, Wataya Y, Tran QK, Kadota S. 2003. In vitro antiplasmodial activity of antimalarial medicinal plants used in Vietnamese traditional medicine. Journal of Ethnopharmacology, 86, 249-252.

154. Ullah M, Khan MU, Mahmood A, Malik RN, Hussain M, Wazir SM, Daud M, Shinwari ZK. 2013. An ethnobotanical survey of indigenous medicinal plants in Wana district South Waziristan agency, Pakistan. Journal of Ethnopharmacology, 150, 918-924.

155. Upadhayay N. 2013. Phytosociological documentation of traditional knowledge of Barleria acanthoides Vahl. International Journal of Science and Research, 5, 1188-1191.

156. Villaescusa L, Diaz AM, Martin T, Gasquet M, Delmas F, Balansard G. 1996. Preliminary screening of antiprotozoal activity of Jasonia glutinosa aerial parts. Pharmaceutical Biology, 34, 303-304.

157. Wabo Pone J, Fossi Tankoua O, Yondo J, Komtangi MC, Mbida M, Bilong Bilong CF. 2011. The in vitro effects of aqueous and ethanolic extracts of the leaves of Ageratum conyzoides (Asteraceae) on three life cycle stages of the parasitic nematode Heligmosomoides bakeri (Nematoda: Heligmosomatidae). Veterinary Medicine International. 140293.

158. Wang G, Han J, Feng T, Li F, Zhu B. 2009. Bioassayguided isolation and identification of active compounds from Fructus arctii against Dactylogyrus intermedius (Monogenea) in goldfish (Carassius auratus). Parasitology Research, 106, 247-255.

159. Wanzala W, Takken W, Mukabana WR, Pala AO, Hassanali A. 2012. Ethnoknowledge of Bukusu community on livestock tick prevention and control in Bungoma district, Western Kenya. Journal of Ethnopharmacology, 140, 298-324.

160. Williams AR, Peña-Espinoza MA, Boas U, Simonsen HT, Enemark HL, Thamsborg SM. 2016. Anthelmintic activity of chicory (Cichorium intybus): In vitro effects on swine nematodes and relationship to sesquiterpene lactone composition. Parasitology, 143, 770-777.

161. Yamamoto ES, Campos BL, Laurenti MD, Lago JH, dos Santos Grecco S, Corbett CE, Passero LF. 2014. Treatment with triterpenic fraction purified from Baccharis uncinella leaves inhibits Leishmania (Leishmania) amazonensis spreading and improves Th1 immune response in infected mice. Parasitology Research, 13, 333-339.

162. Yamamoto ES, Campos BLS, Jesus JA, Laurenti MD, Ribeiro SP, Kallás EG, Rafael-Fernandes M, SantosGomes G, Silva MS, Sessa DP, Lago JHG, Levy D, Passero LFD. 2015. The effect of ursolic acid on Leishmania (Leishmania) amazonensis is related to programed cell death and presents therapeutic potential in experimental cutaneous leishmaniasis. PLoS One, 10, e 0144946.

163. Yildiz K, Basalan M, Duru O, Gokpinar S. 2011. Antiparasitic efficiency of Artemisia absinthium on Toxocara cati in naturally infected cats. Turkish Journal of Parasitology, 35, 10-14.

164. Yu HW, Wright CW, Cai Y, Yang SL, Phillipson JD, Kirby GC, Warhurst DC. 1994. Antiprotozoal activities of Centipeda minima. Phytotherapy Research, 8, 436-438.

165. Zahari Z, Jani NA, Amanah A, Latif MNA, Majid MIA, Adenan MI. Bioassay-guided isolation of a sesquiterpene lactone of deoxyelephantopin from Elephantopus scaber Linn. active on Trypanosome brucei rhodesience. Phytomedicine, 21, 282-285.

166. Zhu F, Qin C, Tao L, Liu X, Shi Z, Ma X, Jia J, Tan Y, Cui C, Lin J, Tan C, Jiang Y, Chen Y. 2011. Clustered patterns of species origins of nature-derived drugs and clues for future bioprospecting. Proceedings of the National Academy of Sciences, 108, 12943-12948.

Cite this article as: Panda SK, Luyten W. 2018. Antiparasitic activity in Asteraceae with special attention to ethnobotanical use by the tribes of Odisha, India. Parasite 25, 10 
O PARASTE

An international open-access, peer-reviewed, online journal publishing high quality papers on all aspects of human and animal parasitology

Reviews, articles and short notes may be submitted. Fields include, but are not limited to: general, medical and veterinary parasitology; morphology, including ultrastructure; parasite systematics, including entomology, acarology, helminthology and protistology, and molecular analyses; molecular biology and biochemistry; immunology of parasitic diseases; host-parasite relationships; ecology and life history of parasites; epidemiology; therapeutics; new diagnostic tools.

All papers in Parasite are published in English. Manuscripts should have a broad interest and must not have been published or submitted elsewhere. No limit is imposed on the length of manuscripts.

Parasite (open-access) continues Parasite (print and online editions, 1994-2012) and Annales de Parasitologie Humaine et Comparée (1923-1993) and is the official journal of the Société Française de Parasitologie. 